Nagoya Math. J., 224(1) (2016), 10-36

DOI $10.1017 / \mathrm{nmj} .2016 .14$

\title{
F-PURITY VERSUS LOG CANONICITY FOR POLYNOMIALS
}

\author{
DANIEL J. HERNÁNDEZ
}

\begin{abstract}
In this article, we consider the conjectured relationship between $F$-purity and $\log$ canonicity for polynomials over $\mathbb{C}$. In particular, we show that $\log$ canonicity corresponds to dense $F$-pure type for all polynomials whose supporting monomials satisfy a certain nondegeneracy condition. We also show that log canonicity corresponds to dense $F$-pure type for very general polynomials over $\mathbb{C}$. Our methods rely on showing that the $F$-pure and log canonical thresholds agree for infinitely many primes, and we accomplish this by comparing these thresholds with the thresholds associated to their monomial term ideals.
\end{abstract}

\section{$\S 1$. Introduction}

Let $R$ denote a polynomial ring over a field of characteristic $p>0$ with homogeneous maximal ideal $\mathfrak{m}$. Given an ideal $\mathfrak{a}$ of $R$ contained in $\mathfrak{m}$, the F-pure threshold of $\mathfrak{a}$ (at the origin) is a numerical invariant measuring the singularities of $\mathfrak{a}$ near the origin, and is closely related to the theory of $F$-purity and tight closure (see [HR76, HH90, HY03]).

In this article, we are particularly interested in the connection between the $F$-pure threshold of a polynomial $f \in \mathfrak{m}$, and the $F$-pure threshold of $\mathfrak{a}_{f}$, the monomial ideal generated by the terms of $f$. The basic relationship between these two invariants, which essentially follows from the containment $f \in \mathfrak{a}_{f}$, is the following upper bound for $\boldsymbol{f p t}(f)$ :

$$
\boldsymbol{f p t}(f) \leqslant \min \left\{1, \boldsymbol{f p t}\left(\mathfrak{a}_{f}\right)\right\} .
$$

This inequality may be strict: if $f=x^{p}+y^{p}$, then $\operatorname{fpt}(f)=\frac{1}{p}$, while $\operatorname{fpt}\left(\mathfrak{a}_{f}\right)=\frac{2}{p}$.

In the first part of this article, we aim to understand when equality holds in (1.1). In Definition 17, we associate to a polynomial $f$ (over a

Received March 23, 2014. Revised September 15, 2015. Accepted June 10, 2015.

2010 Mathematics subject classification. 13A35, 14B05.

The author gratefully acknowledges support by the National Science Foundation via RTG grant number 0502170 at the University of Michigan.

(C) 2016 by The Editorial Board of the Nagoya Mathematical Journal 
field of arbitrary characteristic) a rational polytope $\mathbf{P}$, called the splitting polytope of $f$. The splitting polytope $\mathbf{P}$ is closely related to the familiar Newton polyhedron $\mathbf{N}$ associated to $\mathfrak{a}_{f}$. However, $\mathbf{P}$ and $\mathbf{N}$ differ in the following crucial way: the Newton polyhedron depends only on the ideal $\mathfrak{a}_{f}$ generated by the terms of $f$, while the splitting polytope depends strongly on the terms themselves. For example, $\mathbf{P}$ is always contained in the $n$-fold product of the unit interval (here, $n$ denotes the number of terms of $f$ ), while $\mathbf{N}$ is always a full-dimensional polyhedron in $\mathbb{R}^{m}$ (here, $m$ denotes the number of variables in the ambient polynomial ring). In Section 4, we identify conditions on the Newton polyhedron $\mathbf{N}$, the splitting polytope $\mathbf{P}$, and the characteristic $p$ under which equality holds in (1.1). As these conditions are rather technical, we omit them here, and instead refer the reader to Theorem 42 and Proposition 40.

\subsection{Log canonicity versus $F$-purity}

We now switch gears and consider an invariant of singularities defined over $\mathbb{C}$. Let $S$ be a polynomial ring over $\mathbb{C}$, and let $\mathfrak{a}$ be an ideal contained in the homogeneous maximal ideal of $S$. The log canonical threshold of $\mathfrak{a}$ (at the origin), denoted $\operatorname{lct}(\mathfrak{a})$, is a numerical invariant measuring the singularities of $\mathfrak{a}$ at the origin, and plays an important role in higherdimensional birational geometry (see [BL04, Laz04]). Remarkably, F-pure thresholds can be thought of as the positive characteristic analog of log canonical thresholds (see [Smi00, HW02, HY03, Tak04]). We now sketch the relationship between these two invariants. If $f$ has rational coefficients, one may reduce them modulo $p \gg 0$ to obtain polynomials $f_{p}$ over the finite fields $\mathbb{F}_{p}$. If $f$ has complex (but not necessarily rational) coefficients, a generalization of this method, called the process of reduction to positive characteristic, allows one to produce a family of positive characteristic models $f_{p}$ over finite fields of characteristic $p$. Using the results of [HY03], it is observed in [MTW05] that

$$
\mathbf{f p t}\left(f_{p}\right) \leqslant \operatorname{lct}(f) \text { for } p \gg 0, \text { and that } \lim _{p \rightarrow \infty} \mathbf{f p t}\left(f_{p}\right)=\operatorname{lct}(f) .
$$

We say that log canonicity equals dense (respectively, open) F-pure type for $f$ whenever $\mathbf{f p t}\left(f_{p}\right)=\operatorname{lct}(f)$ for infinitely many $p$ (respectively, for all $p \gg 0$ ) (see Remark 55 for a justification of this terminology). It is conjectured that log canonicity always equals dense $F$-pure type, and verification of this correspondence is a long-standing open problem (see [Fed83, Smi97, EM06]). We now summarize the related results in this article. 
Theorem A. (Cf. Theorem 56) Let $f$ denote a polynomial over $\mathbb{C}$ vanishing at the origin. If the supporting monomials of $f$ satisfy a certain nondegeneracy condition, then there exists a simple characterization of the set of all primes $p$ for which $\mathbf{f} \mathbf{p t}\left(f_{p}\right)=\mathbf{l} \mathbf{c t}(f)$. Moreover, this characterization shows that log canonicity equals dense F-pure type for $f$.

For the precise statement (including the description of the nondegeneracy condition, which may be stated in terms of the associated splitting polytope $\mathbf{P}$ and Newton polyhedra $\mathbf{N}$ ), we refer the reader to Theorem 56 . We note that the techniques used in proving Theorem 56 are related to those used by Shibuta and Takagi in their calculation of log canonical thresholds of binomial ideals appearing in [ST09].

TheOrem B. (Cf. Theorem 58) Let $f$ be a polynomial over $\mathbb{C}$ vanishing at the origin. If the coefficients appearing in the terms of $f$ form an algebraically independent sequence over $\mathbb{Q}$, then log canonicity equals dense F-pure type for $f$.

These theorems differ in an important way: the first requires some nondegeneracy of the supporting monomials of $f$, but assumes nothing about the coefficients of $f$, while the second requires some generality of the coefficients of $f$, but assumes nothing about the supporting monomials. Moreover, under certain conditions, the first theorem characterizes the set of primes for which the $F$-pure and log canonical thresholds agree, while the second provides no such characterization.

We now outline our method for establishing these results. Let $f$ denote a polynomial over $\mathbb{C}$, and let $\mathfrak{a}_{f}$ denote its term ideal. An important component of our argument relies on the fact that the characteristic zero analog of (1.1) holds, that is, on the fact that

$$
\operatorname{lct}(f) \leqslant \min \left\{1, \operatorname{lct}\left(\mathfrak{a}_{f}\right)\right\} .
$$

Let $f_{p}$ denote the reduction of $f$ to characteristic $p \gg 0$. For such $p$, the monomials appearing in $f$ and $f_{p}$ agree, so that $\mathfrak{a}_{f}$ and $\mathfrak{a}_{f_{p}}$ (the term ideals of $f$ and $f_{p}$ ) are generated by the same monomials. Moreover, it follows from [HY03] that $\mathbf{f p t}\left(\mathfrak{a}_{f_{p}}\right)=\operatorname{lct}\left(\mathfrak{a}_{f}\right)$ for $p \gg 0$. Using results referenced earlier in this introduction (i.e., Theorem 42 and Proposition 40), we are able to show that $\mathbf{f p t}\left(f_{p}\right)=\min \left\{1, \mathbf{f p t}\left(\mathfrak{a}_{f_{p}}\right)\right\}$ for infinitely (or all but finitely) many $p$, and an application of the relations in (1.2) shows that for such $p$,

$$
\mathbf{f p t}\left(f_{p}\right) \leqslant \operatorname{lct}(f) \leqslant \min \left\{1, \boldsymbol{l c t}\left(\mathfrak{a}_{f}\right)\right\}=\min \left\{1, \boldsymbol{f p t}\left(\mathfrak{a}_{f_{p}}\right)\right\}=\mathbf{f p t}\left(f_{p}\right),
$$

forcing equality throughout. 


\section{$\S 2$. Base $p$ expansions}

Definition 1. Let $\lambda$ be a nonzero real number in the unit interval. The nonterminating base $p$ expansion of $\lambda$ is the unique expression $\lambda=\sum_{e \geqslant 1} \lambda^{(e)} \cdot p^{-e}$ with the property that the integers $0 \leqslant \lambda^{(e)} \leqslant p-1$ are all not eventually zero. The number $\lambda^{(e)}$ is called the $e$ th digit of $\lambda$ in base $p$. By convention, we set $\lambda^{(0)}=0^{(e)}=0$.

Definition 2. Let $\lambda$ be a nonzero real number in the unit interval, and fix a prime $p$. Given an integer $e \geqslant 1$, we call $\langle\lambda\rangle_{e}:=\sum_{s=1}^{e} \lambda^{(s)} \cdot p^{-s}$ the eth truncation of $\lambda$ in base $p$. By convention, we set $\langle\lambda\rangle_{0}=\langle 0\rangle_{e}=0$. Given a vector $\boldsymbol{\lambda} \in[0,1]^{n}$, we use $\langle\boldsymbol{\lambda}\rangle_{e}$ to denote the vector whose entries are the $e$ th truncations of the entries of $\boldsymbol{\lambda}$.

When $p$ is obvious from the context (e.g., the characteristic of an ambient field), we omit any reference to the base when discussing digits and truncations.

Lemma 3. If $\left(p^{e}-1\right) \cdot \lambda \in \mathbb{N}$ for some $e$, then $\left(p^{e}-1\right) \cdot \lambda=p^{e} \cdot\langle\lambda\rangle_{e}$.

Proof. This follows from the observation (whose verification is left to the reader) that if $\left(p^{e}-1\right) \cdot \lambda \in \mathbb{N}$, then the digits of $\lambda$ (in base $p$ ) are periodic and repeat after $e$ terms.

LEMmA 4. If $\lambda \in(0,1]$, then $p^{e} \cdot\langle\lambda\rangle_{e} \in \mathbb{N}$, and $\langle\lambda\rangle_{e}<\lambda \leqslant\langle\lambda\rangle_{e}+\frac{1}{p^{e}}$. Furthermore, if $(p-1) \cdot \lambda \in \mathbb{N}$, then $\lambda^{(e)}=(p-1) \cdot \lambda$ for every $e \geqslant 1$.

Proof. The first two assertions follow from the definitions. For the third, note that the nonterminating expansion for $\lambda$ can be obtained by multiplying each term in the expansion $1=\sum_{e \geqslant 1}(p-1) \cdot p^{-e}$ by $\lambda$.

Definition 5. Let $\left(\lambda_{1}, \ldots, \lambda_{n}\right) \in[0,1]^{n}$. We say that $\lambda_{1}, \ldots, \lambda_{n}$ add without carrying (base $p$ ) if $\lambda_{1}{ }^{(e)}+\cdots+\lambda_{n}{ }^{(e)} \leqslant p-1$ for every $e \geqslant 1$.

The notion of adding without carrying is relevant in light of the following classical result.

Lemma 6. [Dic02, Luc78] Fix $\mathbf{k}=\left(k_{1}, \ldots, k_{n}\right) \in \mathbb{N}^{n}$, and set $N=k_{1}+$ $\cdots+k_{n}$. For every $i$, let $k_{i}=\sum_{e \geqslant 0} k_{i, e} \cdot p^{e}$ denote the (unique) base $p$ expansion of the integer $k_{i}$ (so that $k_{i, e}=0$ for $e \gg 0$ ), and, similarly, let $N=\sum_{e \geqslant 0} N_{e} \cdot p^{e}$ denote the (unique) base $p$ expansion of $N$. Then, the multinomial coefficient $\left(\begin{array}{l}N \\ \mathbf{k}\end{array}\right):=\frac{N !}{k_{1} ! \cdots k_{n} !} \not \equiv 0 \bmod p$ if and only if $k_{1, e}+\cdots+$ $k_{n, e}=N_{e}$ for every $e \geqslant 0$. 
COROllary 7. The entries of a vector $\left(\lambda_{1}, \ldots, \lambda_{n}\right) \in[0,1]^{n}$ add without carrying if and only if $\left(\begin{array}{c}p^{e}\left\langle\lambda_{1}\right\rangle_{e}+\cdots+p^{e}\left\langle\lambda_{n}\right\rangle_{e} \\ p^{e}\left\langle\lambda_{1}\right\rangle_{e}, \ldots, p^{e}\left\langle\lambda_{n}\right\rangle_{e}\end{array}\right) \neq \equiv \bmod p$ for every $e \geqslant 1$ (or, equivalently, for infinitely many $e \geqslant 1$ ).

Proof. Lemma 6 implies that $\left(\begin{array}{c}p^{e}\left\langle\lambda_{1}\right\rangle_{e}+\cdots+p^{e}\left\langle\lambda_{n}\right\rangle_{e} \\ p^{e}\left\langle\lambda_{1}\right\rangle_{e}, \ldots, p^{e}\left\langle\lambda_{n}\right\rangle_{e}\end{array}\right) \not \equiv 0 \bmod p \Longleftrightarrow \lambda_{1}{ }^{(s)}+$ $\cdots+\lambda_{n}{ }^{(s)} \leqslant p-1$ for every $1 \leqslant s \leqslant e$, and the claim follows.

LEMma 8. $\operatorname{Let}\left(\lambda_{1}, \ldots, \lambda_{n}\right) \in \mathbb{Q}^{n} \cap[0,1]^{n}$.

(1) If $\lambda_{1}+\cdots+\lambda_{n} \leqslant 1$, then $\lambda_{1}, \ldots, \lambda_{n}$ add without carrying for infinitely many $p$.

(2) If $\lambda_{1}+\cdots+\lambda_{n}>1$, then $\lambda_{1}{ }^{(1)}+\cdots+\lambda_{n}{ }^{(1)} \geqslant p$ whenever $p \gg 0$.

Proof. By Dirichlet's theorem, $(p-1) \cdot\left(\lambda_{1}, \ldots, \lambda_{n}\right) \in \mathbb{N}^{n}$ for infinitely many $p$. For such $p$, Lemma 4 shows that $\lambda_{i}^{(e)}=(p-1) \cdot \lambda_{i}$ for each $i$ and $e$, and therefore $\sum \lambda_{i}{ }^{(e)}=(p-1) \cdot \sum \lambda_{i}$, which establishes the first point. For the second point, note that

$$
\lambda_{i}^{(1)}=p\left\langle\lambda_{i}\right\rangle_{1}=p \cdot \lambda_{i}-p \cdot\left(\lambda_{i}-\left\langle\lambda_{i}\right\rangle_{1}\right) \geqslant p \cdot \lambda_{i}-1
$$

where the last inequality follows from Lemma 4. Consequently,

$$
\sum_{i=1}^{n} \lambda_{i}{ }^{(1)} \geqslant p \cdot \sum_{i=1}^{n} \lambda_{i}-n=p+p \cdot\left(\sum_{i=1}^{n} \lambda_{i}-1\right)-n
$$

and, hence, $\sum \lambda_{i}^{(1)} \geqslant p$ whenever $p \cdot\left(\sum \lambda_{i}-1\right) \geqslant n$.

\section{§3. F-pure thresholds}

Let $R$ denote a polynomial ring over a field of characteristic $p>0$, and let $\mathfrak{m}=\left(x_{1}, \ldots, x_{m}\right)$ denote the homogeneous maximal ideal of $R$. For every ideal $I$ of $R$, let $I^{\left[p^{e}\right]}$ denote the ideal generated by the set $\left\{f^{p^{e}}: f \in I\right\}$. We call $I^{\left[p^{e}\right]}$ the eth Frobenius power of $I$.

Definition 9. [TW04, MTW05] Fix a nonzero ideal $\mathfrak{a} \subseteq \mathfrak{m}$ generated by $t$ elements. By the pigeon-hole principle, we have that $\mathfrak{a}^{t\left(p^{e}-1\right)+1} \subseteq \mathfrak{m}^{\left[p^{e}\right]}$, and hence

$$
\nu_{\mathfrak{a}}\left(p^{e}\right)=\max \left\{w: \mathfrak{a}^{w} \notin \mathfrak{m}^{\left[p^{e}\right]}\right\}
$$

is a well-defined integer, bounded above by $t\left(p^{e}-1\right)+1$. Thus, the sequence $\left\{p^{-e} \cdot \nu_{\mathfrak{a}}\left(p^{e}\right)\right\}$ is bounded above by $t$, and the flatness of the Frobenius 
morphism can be used to show that this sequence is nondecreasing, so that the limit

$$
\mathbf{f p t}(\mathfrak{a})=\lim _{e \rightarrow \infty} p^{-e} \cdot \nu_{\mathfrak{a}}\left(p^{e}\right)
$$

exists. We call this limit the $F$-pure threshold of $\mathfrak{a}$ at the origin. In the case that $\mathfrak{a}$ is a principal ideal generated by a polynomial $f \in \mathfrak{m}$, we write $\operatorname{fpt}(f)$ instead of $\mathbf{f p t}(\mathfrak{a})$.

REMARK 10. Although it is not obvious from the definition, the $F$-pure threshold is a rational number (see [BMS08, Theorem 3.1]).

REMARK 11. As $\mathfrak{a} \neq 0$, we have that $\mathfrak{a} \nsubseteq \mathfrak{m}^{\left[p^{e}\right]}$ for large values of $e$, so that $\nu_{\mathfrak{a}}\left(p^{e}\right) \neq 0$ for $e \gg 0$, and hence $\mathbf{f p t}(\mathfrak{a}) \neq 0$. Moreover, the bound $\nu_{\mathfrak{a}}\left(p^{e}\right) \leqslant t\left(p^{e}-1\right)+1$ shows that $\mathbf{f p t}(\mathfrak{a}) \leqslant t$. In particular, when $\mathfrak{a}=(f)$ is principal, we have that $\mathbf{f p t}(f) \in(0,1]$.

REMARK 12. If $\mathfrak{a} \subseteq \mathfrak{b}$ are ideals contained in $\mathfrak{m}$, then $\nu_{\mathfrak{a}}\left(p^{e}\right) \leqslant \nu_{\mathfrak{b}}\left(p^{e}\right)$, and hence $\mathbf{f p t}(\mathfrak{a}) \leqslant \boldsymbol{f p t}(\mathfrak{b})$. In particular, if $f \in \mathfrak{m}$ is a polynomial and $\mathfrak{a}_{f}$ is its term ideal, then

$$
\mathbf{f p t}(f) \leqslant \min \left\{1, \boldsymbol{f p t}\left(\mathfrak{a}_{f}\right)\right\} .
$$

The following lemma, which is well known to experts, plays a key role in this article.

LEMmA 13. Let $\lambda \in(0,1]$ be a rational number such that $\left(p^{e}-1\right) \cdot \lambda \in \mathbb{N}$ for some $e \geqslant 1$. Then, $\mathbf{f p t}(f) \geqslant \lambda$ if and only if $f^{\left(p^{e}-1\right) \cdot \lambda} \notin \mathfrak{m}^{\left[p^{e}\right]}$.

Proof. This may be deduced, for example, from either [Sch08, Proposition 3.3] and [Sch08, Lemma 5.2], [Her12, Key Lemma 3.1] and [Her12, Proposition 4.2], or [MTW05, Proposition 1.9] and [BMS09, Proposition 4.3(ii)]. For the convenience of the reader, we present a proof based on the last of these options (as it only involves concepts introduced herein), and we begin by recalling the relevant facts. In what follows, we fix an integer $e \geqslant 1$.

(1) $\nu_{f}\left(p^{e}\right)=\left\lceil p^{e} \cdot \mathbf{f p t}(f)\right\rceil-1$ (see [MTW05, Proposition 1.9]).

(2) If $a \in \mathbb{N}$, and if $\mathbf{f p t}(f)>\frac{a}{p^{e}}$, then $\mathbf{f p t}(f) \geqslant \frac{a}{p^{e}-1}$ (see [BMS09, Proposition 4.3(ii)]).

Note that, as $\lceil\alpha\rceil<\alpha+1$ for every real number $\alpha$, (1) above implies that (3) $\nu_{f}\left(p^{e}\right)<p^{e} \cdot \mathbf{f p t}(f)$. 
We now begin our proof. First, note that the assumption that $\left(p^{e}-1\right) \lambda \in$ $\mathbb{N}$ implies that

$$
\left\lceil p^{e} \lambda\right\rceil=\left\lceil p^{e} \lambda-\lambda+\lambda\right\rceil=\left(p^{e}-1\right) \lambda+\lceil\lambda\rceil=\left(p^{e}-1\right) \lambda+1
$$

If $\boldsymbol{f p t}(f) \geqslant \lambda$, then combining (3.1) and (1) above shows that

$$
\nu_{f}\left(p^{e}\right)=\left\lceil p^{e} \cdot \mathbf{f p t}(f)\right\rceil-1 \geqslant\left\lceil p^{e} \lambda\right\rceil-1=\left(p^{e}-1\right) \lambda .
$$

On the other hand, if $\left(p^{e}-1\right) \cdot \lambda \leqslant \nu_{f}\left(p^{e}\right)$, then combing this with (3) above implies that

$$
\frac{\left(p^{e}-1\right) \cdot \lambda}{p^{e}} \leqslant \frac{\nu_{f}\left(p^{e}\right)}{p^{e}}<\operatorname{fpt}(f),
$$

and (2) above then shows that $\mathbf{f p t}(f) \geqslant \frac{\left(p^{e}-1\right) \cdot \lambda}{p^{e}-1}=\lambda$.

In summary, we have just shown that $\mathbf{f p t}(f) \geqslant \lambda$ if and only if $\nu_{f}\left(p^{e}\right) \geqslant$ $\left(p^{e}-1\right) \cdot \lambda$, and the claim then follows.

\section{§4. Polyhedral constructions}

In this section, we consider two key polyhedra associated to polynomials, and we begin by recalling some basic notions from convex geometry. Throughout this discussion, our main reference is [Web94].

\subsection{Polyhedral sets}

Given a linear form $L \in \mathbb{R}\left[z_{1}, \ldots, z_{m}\right]$ and a real number $\beta$, we use $\mathrm{H}_{\beta}^{L}$ to denote the hyperplane $\mathrm{H}_{\beta}^{L}=\left\{\mathrm{v} \in \mathbb{R}^{m}: L(\mathrm{v})=\beta\right\}$, and we use $\overline{\mathrm{HS}}_{\beta}^{L}$ to denote the closed upper halfspace $\overline{\mathrm{HS}}_{\beta}^{L}=\left\{\mathrm{v} \in \mathbb{R}^{m}: L(\mathrm{v}) \geqslant \beta\right\}$. Given $\mathscr{P} \subseteq \mathbb{R}^{m}, \overline{\mathrm{HS}}_{\beta}^{L}$ is called a supporting halfspace of $\mathscr{P}$ if $\mathscr{P} \subseteq \overline{\mathrm{HS}}_{\beta}^{L}$ and $\mathrm{H}_{\beta}^{L} \cap \mathscr{P} \neq \emptyset$. In this case, $\mathrm{H}_{\beta}^{L} \cap \mathscr{P}$ is called an (exposed) face of $\mathscr{P}$. Recall that a subset $\mathscr{P} \subseteq \mathbb{R}^{m}$ is called a polyhedral set if there exist finitely many linear forms $L_{1}, \ldots L_{d}$ in $\mathbb{R}\left[z_{1}, \ldots, z_{m}\right]$ and elements $\beta_{1}, \ldots, \beta_{d} \in \mathbb{R}$ such that

$$
\mathscr{P}=\overline{\mathrm{HS}}_{\beta_{1}}^{L_{1}} \cap \cdots \cap \overline{\mathrm{HS}}_{\beta_{d}}^{L_{d}}
$$

If we may choose the linear forms $L_{1}, \ldots, L_{d}$ to have rational coefficients, and the numbers $\beta_{1}, \ldots \beta_{d}$ to be rational, then we call the polyhedral set $\mathscr{P}$ rational. In the case that a polyhedral set is rational, all of its faces may described by rational data. A compact polyhedral set is called a polytope. 
Given any subset $\mathscr{C} \subseteq \mathbb{R}^{m}$, we use cone $(\mathscr{C})$ to denote the collection of all finite sums $\sum_{\mathbf{v}} \lambda_{\mathbf{v}} \cdot \mathbf{v}$ with $\mathrm{v} \in \mathscr{C}$ such that $\lambda_{\mathbf{v}} \geqslant 0$; we call cone $(\mathscr{C})$ the cone generated by $\mathscr{C}$. Given $\mathbf{v} \in \mathbb{R}^{m}$, we call cone $(\{\mathbf{v}\})$ the ray generated by $\mathbf{v}$. We also use convex $(\mathscr{C})$ to denote the collection of all finite sums $\sum_{\mathbf{v}} \lambda_{\mathbf{v}} \cdot \mathbf{v}$ with $\mathrm{v} \in \mathscr{C}$ such that $\lambda_{\mathbf{v}} \geqslant 0$ and $\sum_{\mathbf{v}} \lambda_{\mathbf{v}}=1$; we call convex $(\mathscr{C})$ the convex hull of $\mathscr{C}$. Although it is not obvious from these definitions, both cone $(\mathscr{C})$ and convex $(\mathscr{C})$ are rational polyhedral sets whenever $\mathscr{C} \subseteq \mathbb{Q}^{m}$ (see [Web94, Theorems 4.1.1 and 3.2.5]). Given two subsets $V$ and $W$ of $\mathbb{R}^{m}$, we use $V+W$ to denote $\{\mathbf{v}+\mathbf{w}: \mathbf{v} \in V, \mathbf{w} \in W\}$, the Minkowski sum of $V$ and $W$. We conclude by recalling the following structural result (often called the finite-basis theorem for polyhedra): a set in $\mathbb{R}^{m}$ is polyhedral if and only if it is of the form convex $(\mathscr{C})+\operatorname{cone}(\mathscr{D})$, where $\mathscr{C}$ and $\mathscr{D}$ are two finite (and possibly empty) subsets of $\mathbb{R}^{m}$, and is rational if and only if $\mathscr{C}$ and $\mathscr{D}$ are contained in $\mathbb{Q}^{m}$ (see [Web94, Theorem 4.1.2]).

REMARK 14. By the finite-basis theorem for polyhedra, every polyhedron $\mathscr{P} \subseteq \mathbb{R}^{m}$ is of the form convex $(\mathscr{C})+\operatorname{cone}(\mathscr{D})$ for some finite subsets $\mathscr{C}, \mathscr{D} \subseteq \mathbb{R}_{\geqslant 0}^{m}$. It then follows from the finiteness of $\mathscr{C}$ (and, hence, the boundedness of convex $(\mathscr{C})$ ) that the polyhedron $\mathscr{P}$ is unbounded if and only if it contains a ray of the form $\mathbf{v}+\lambda \cdot \mathbf{w}$, with $\mathbf{v} \in \mathscr{P}$, and $\mathbf{0} \neq \mathbf{w} \in \mathbb{R}^{m}$.

\subsection{Splitting polytopes}

Notation 15. For $\mathbf{s}=\left(s_{1}, \ldots, s_{n}\right) \in \mathbb{R}^{n},|\mathbf{s}|$ denotes the coordinate sum $s_{1}+\cdots+s_{n}$. We stress that $|\cdot|$ is not the usual Euclidean norm on $\mathbb{R}^{n}$. Furthermore, when dealing with elements of $\mathbb{R}^{n}$, we use $\prec$ and $\preccurlyeq$ to denote component-wise (strict) inequality. Finally, $\mathbf{1}_{m}$ denotes the element $(1, \ldots, 1) \in \mathbb{R}^{m}$.

Notation 16. Fix a polynomial ring $\mathbb{L}\left[x_{1}, \ldots, x_{m}\right]$ over a field of arbitrary characteristic. Given a vector $\mathbf{a}=\left(a_{1}, \ldots, a_{m}\right) \in \mathbb{N}^{m}$, we use $x^{\mathbf{a}}$ to denote the monomial $x_{1}^{a_{1}} \cdots x_{m}^{a_{m}}$. If $f=\sum_{i=1}^{n} u_{i} \cdot x^{\mathbf{a}_{i}}$ is the unique expression of a polynomial $f$ as an $\mathbb{L}^{*}$-linear combination of distinct monomials, then we use $\operatorname{Supp}(f)$ to denote $\left\{x^{\mathbf{a}_{1}}, \ldots, x^{\mathbf{a}_{n}}\right\}$, the collection of supporting monomials of $f$. We often abuse notation by identifying monomials with their exponent vectors, and thus often write $\operatorname{Supp}(f)=\left\{\mathbf{a}_{1}, \ldots, \mathbf{a}_{n}\right\}$. We continue to use $\mathfrak{a}_{f}$ to denote the term ideal of $f$, that is, the monomial ideal generated by $\operatorname{Supp}(f)$ in $\mathbb{L}\left[x_{1}, \ldots, x_{m}\right]$.

DeFinition 17. Fix an arbitrary field $\mathbb{L}$, and consider a polynomial $f \in$ $\mathbb{L}\left[x_{1}, \ldots, x_{m}\right]$ vanishing at the origin, with $\operatorname{Supp}(f)=\left\{\mathbf{a}_{1}, \ldots, \mathbf{a}_{n}\right\} \subseteq \mathbb{N}^{m}$. 

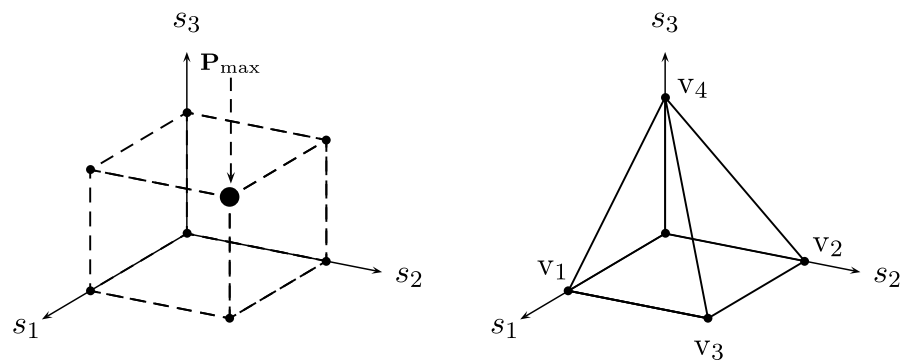

Figure 1.

The splitting polytopes from Examples 20 and 21.

(1) We call the $m \times n$ matrix $\mathbf{E}:=\left[\mathbf{a}_{1} \cdots \mathbf{a}_{n}\right]$ the exponent matrix of $f$.

(2) We call $\mathbf{P}:=\left\{\mathbf{s} \in \mathbb{R}_{\geqslant 0}^{n}: \mathbf{E s} \preccurlyeq \mathbf{1}_{m}\right\}$ the splitting polytope of $f$.

REMARK 18. The exponent matrix and splitting polytope associated to $f$ depend only on $\operatorname{Supp}(f)$. Moreover, it follows by definition that $\mathbf{P}$ is a polyhedral set, and as $\mathbf{E}$ has nonnegative integer entries, $\mathbf{P}$ is a compact subset (and, hence, polytope) in $[0,1]^{n}$.

Definition 19. Consider the linear functional $\mathbb{R}^{n} \rightarrow \mathbb{R}$ given by $\mathbf{s} \mapsto$ $|\mathbf{s}|=s_{1}+\cdots+s_{n}$. As $\mathbf{P}$ is compact, $\beta=\max \{|\mathbf{s}|: \mathbf{s} \in \mathbf{P}\}$ is well defined, and the nonempty set $\{\mathbf{s} \in \mathbf{P}:|\mathbf{s}|=\beta\}$ defines an exposed face of $\mathbf{P}$. We call this face the maximal face of $\mathbf{P}$, and denote it by $\mathbf{P}_{\max }$.

ExAmple 20. If $f=x_{1}^{d_{1}}+\cdots+x_{m}^{d_{m}}$, then $\quad \mathbf{P}=\left\{\mathbf{s} \in \mathbb{R}^{m}: \mathbf{0} \preccurlyeq \mathbf{s} \preccurlyeq\right.$ $\left.\left(1 / d_{1}, \ldots, 1 / d_{m}\right)\right\}$, and $\mathbf{P}_{\max }$ consists of the single point $\left(1 / d_{1}, \ldots, 1 / d_{m}\right)$.

The splitting polytopes considered in the following examples are depicted in figure 1.

ExAmple 21. If $f=x^{a}+y^{b}+x^{c} y^{c}$, then $\mathbf{P}=\left\{\mathbf{s} \succcurlyeq 0:\left[\begin{array}{lll}a & 0 & c \\ 0 & b & c\end{array}\right] \mathbf{s} \preccurlyeq\left[\begin{array}{l}1 \\ 1\end{array}\right]\right\}$ is the convex hull of $\mathrm{v}_{1}=(1 / a, 0,0), \mathrm{v}_{2}=(0,1 / b, 0), \mathrm{v}_{3}=(1 / a, 1 / b, 0)$, $\mathrm{v}_{4}=(0,0,1 / c)$, and $\mathbf{0}$. The description of $\mathbf{P}_{\max }$ depends on the relationship between $a, b$, and $c$.

(1) If $1 / a+1 / b>1 / c$, then $\mathbf{P}_{\max }=\left\{\mathrm{v}_{3}\right\}$.

(2) If $1 / a+1 / b<1 / c$, then $\mathbf{P}_{\max }=\left\{\mathrm{v}_{4}\right\}$.

(3) If $1 / a+1 / b=1 / c$, then $\mathbf{P}_{\max }$ is the line segment connecting $\mathrm{v}_{3}$ and $\mathrm{v}_{4}$.

Definition 22. We call an element of $\mathbf{P}_{\max }$ a maximal point, and we say that $\mathbf{P}$ contains a unique maximal point if ${ }^{\#} \mathbf{P}_{\max }=1$. 
Example 23. In Example 20, $\mathbf{P}$ always has a unique maximal point. In Example 21, we see that $\mathbf{P}$ contains a unique maximal point if and only if $1 / a+1 / b \neq 1 / c$.

We conclude this discussion by illustrating an important property of polynomials whose splitting polytope contains a unique maximal point. We refer the reader to Remark 34 for a description of this condition in terms of Newton polyhedra.

Lemma 24. Suppose that $\mathbf{P}$ has a unique maximal point $\boldsymbol{\eta} \in \mathbf{P}$.

(1) If $|\mathbf{s}|=\left|\langle\boldsymbol{\eta}\rangle_{e}\right|$ and $\mathbf{E s}=\mathbf{E}\langle\boldsymbol{\eta}\rangle_{e}$ for some $\mathbf{s} \in \mathbb{R}_{\geqslant 0}^{n}$, then $\mathbf{s}=\langle\boldsymbol{\eta}\rangle_{e}$.

(2) If $|\mathbf{s}|=|\boldsymbol{\nu}|$, Es $=\mathbf{E} \boldsymbol{\nu}$, and $\boldsymbol{\nu} \preccurlyeq\langle\boldsymbol{\eta}\rangle_{\text {e }}$ for some $\boldsymbol{\nu}, \mathbf{s}$ in $\mathbb{R}_{\geqslant 0}^{n}$, then $\mathbf{s}=\boldsymbol{\nu}$.

Proof. To prove the first statement, let $\boldsymbol{\eta}^{\prime}:=\mathbf{s}+\boldsymbol{\eta}-\langle\boldsymbol{\eta}\rangle_{e}$. By hypothesis, $\boldsymbol{\eta}^{\prime} \succcurlyeq \mathbf{s} \succcurlyeq \mathbf{0}, \mathbf{E} \boldsymbol{\eta}^{\prime}=\mathbf{E s}+\mathbf{E} \boldsymbol{\eta}-\mathbf{E}\langle\boldsymbol{\eta}\rangle_{e}=\mathbf{E} \boldsymbol{\eta}$, and $\left|\boldsymbol{\eta}^{\prime}\right|=|\mathbf{s}|+|\boldsymbol{\eta}|-\left|\langle\boldsymbol{\eta}\rangle_{e}\right|=|\boldsymbol{\eta}|$, which shows that $\boldsymbol{\eta}^{\prime}$ is a maximal point of $\mathbf{P}$. Thus, $\boldsymbol{\eta}^{\prime}=\boldsymbol{\eta}$, and $\mathbf{s}=\langle\boldsymbol{\eta}\rangle_{e}$.

For the second statement, let $\mathbf{s}^{\prime}:=\mathbf{s}+\langle\boldsymbol{\eta}\rangle_{e}-\boldsymbol{\nu}$. By hypothesis, $\mathbf{s}^{\prime} \succcurlyeq \mathbf{0}$, $\left|\mathbf{s}^{\prime}\right|=\left|\langle\boldsymbol{\eta}\rangle_{e}\right|$, and $\mathbf{E s}^{\prime}=\mathbf{E}\langle\boldsymbol{\eta}\rangle_{e}$. The first statement, applied to $\mathbf{s}^{\prime}$, shows that $\mathbf{s}^{\prime}=\langle\boldsymbol{\eta}\rangle_{e}$, and thus $\mathbf{s}=\boldsymbol{\nu}$.

Corollary 25. Fix a field $\mathbb{L}$ of characteristic $p$, and a polynomial $f=\sum_{i=1}^{n} u_{i} \cdot x^{\mathbf{a}_{i}}$ in $\mathbb{L}\left[x_{1}, \ldots, x_{m}\right]$ vanishing at the origin, and such that the associated splitting polytope $\mathbf{P}$ has a unique maximal point $\boldsymbol{\eta} \in \mathbf{P}$.

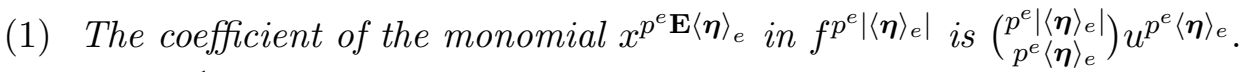

(2) If $\boldsymbol{\nu} \in \frac{1}{p^{e}} \cdot \mathbb{N}^{n}$ is an index such that $\boldsymbol{\nu} \preccurlyeq\langle\boldsymbol{\eta}\rangle_{e}$, then the coefficient of the monomial $\boldsymbol{x}^{p^{e} \mathbf{E} \boldsymbol{\nu}}$ in $f^{p^{e}|\boldsymbol{\nu}|}$ is $\left(\begin{array}{c}p^{e}|\boldsymbol{\nu}| \\ p^{e} \boldsymbol{\nu}\end{array}\right) u^{p^{e} \boldsymbol{\nu}}$.

Proof. This follows immediately from Lemma 24, and the multinomial theorem.

\subsection{Newton polyhedra}

Definition 26. Fix an arbitrary field $\mathbb{L}$, and consider a polynomial $f \in$ $\mathbb{L}\left[x_{1}, \ldots, x_{m}\right]$ vanishing at the origin. We call $\mathbf{N}:=\operatorname{convex}(\operatorname{Supp}(f))+\mathbb{R}_{\geqslant 0}^{m}$ the Newton polyhedron of $f$.

REMARK 27. It follows from the discussion in Section 4.1 that $\mathbf{N}$ is, indeed, a polyhedron. Moreover, since $\operatorname{Supp}(f) \subseteq \mathbb{N}^{m}$ and $\mathbb{R}_{\geqslant 0}^{m}$ is the cone generated by the standard basis elements of $\mathbb{Q}^{m}$, it follows that $\mathbf{N}$ is rational.

LEMMA 28. If $\mathbf{N}$ is the Newton polyhedron of $f \in \mathbb{L}\left[x_{1}, \ldots, x_{m}\right]$, then every nonempty face of $\mathbf{N}$ is of the form $H_{\beta}^{L} \cap \mathbf{N}$, where $\beta \in \mathbb{Q}_{\geqslant 0}$, and 
$L \in \mathbb{Q}\left[z_{1}, \ldots, z_{m}\right]$ is a $\mathbb{Q} \geqslant 0$-linear combination of the variables. Furthermore, such a face is bounded if and only if $L$ is a $\mathbb{Q}_{>0}$-linear combination of the variables (in which case, $\beta \in \mathbb{Q}_{>0}$ ).

Proof. By Remark 27, every face of $\mathbf{N}$ is of the form $\mathrm{H}_{\beta}^{L} \cap \mathbf{N}$, where $L=\beta_{1} z_{1}+\cdots+\beta_{m} z_{n}$, and $\beta, \beta_{1}, \ldots, \beta_{m} \in \mathbb{Q}$. Fix $\mathrm{v} \in \mathrm{H}_{\beta}^{L} \cap \mathbf{N}$. By definition, $\mathrm{v}+\lambda \cdot \mathbf{e}_{i} \in \mathbf{N}$ for every $\lambda>0$, and so

$$
\beta \leqslant L\left(\mathrm{v}+\lambda \cdot \mathbf{e}_{i}\right)=L(\mathrm{v})+\lambda \cdot L\left(\mathbf{e}_{i}\right)=\beta+\lambda \cdot \beta_{i} .
$$

As $\lambda>0,(4.1)$ implies $\beta_{i} \geqslant 0$. Furthermore, as we have shown that $L$ is a nonnegative rational combination of the variables, it follows that $L(\mathbf{N}) \subseteq$ $L\left(\mathbb{R}_{\geqslant 0}^{m}\right) \subseteq \mathbb{R}_{\geqslant 0}$. Consequently, as we are assuming that the face $\mathrm{H}_{\beta}^{L} \cap \mathbf{N}=$ $\{\mathrm{v} \in \mathbf{N}: L(\mathrm{v})=\beta\}$ is nonempty, it follows that $\beta$ must be nonnegative. We now prove the second assertion. By Remark 14, we have that $\mathrm{H}_{\beta}^{L} \cap \mathbf{N}$ is bounded if and only if for every $\mathrm{v} \in \mathrm{H}_{\beta}^{L} \cap \mathbf{N}$, and nonzero element $\mathbf{w} \in$ $\mathbb{R}_{\geqslant 0}^{m}$, there exists a positive real number $\lambda$ such that $\mathrm{v}+\lambda \cdot \mathbf{w} \notin \mathrm{H}_{\beta}^{L} \cap \mathbf{N}$. Writing $\mathbf{w}=\sum_{i=1}^{m} w_{i} \cdot \mathbf{e}_{i}$, the preceding condition may be written as $\beta<$ $L(\mathrm{v}+\lambda \cdot \mathbf{w})=L(\mathrm{v})+\sum_{i=1}^{m} \lambda w_{i} \cdot \beta_{i}=\beta+\sum_{i=1}^{m} \lambda w_{i} \cdot \beta_{i}$. As $\lambda>0$, and as $\mathbf{w}$ can be chosen arbitrarily, we conclude that $\mathrm{H}_{\beta}^{L} \cap \mathbf{N}$ is bounded if and only if $\beta_{1}, \ldots, \beta_{m}>0$. Finally, it follows from the positivity of the coefficients of $L$, along with the fact that every point in $\mathbf{N}$ is has nonnegative entries (and at least one positive entry), that $L(\mathbf{N}) \subseteq \mathbb{R}_{>0}$, and hence that $\beta>0$.

Definition 29. By definition, the ray generated by $\mathbf{1}_{m}$ intersects the boundary of $\mathbf{N}$. By [Web94, Theorem 3.2.2], this boundary is the union of the exposed faces of $\mathbf{N}$, and we say that $\mathbf{N}$ is in diagonal position if the ray generated by $\mathbf{1}_{m}$ intersects a bounded face of $\mathbf{N}$.

ExAmPle 30. The polyhedra appearing in Examples 20 and 21 are in diagonal position.

\subsection{Connections}

In this subsection, we fix a field $\mathbb{L}$, a polynomial $f \in \mathbb{L}\left[x_{1}, \ldots, x_{m}\right]$ vanishing at the origin, and distinct, nonzero vectors such that $\operatorname{Supp}(f)=$ $\left\{\mathbf{a}_{1}, \ldots, \mathbf{a}_{n}\right\}$.

LEMma 31. If $\mathbf{P}$ and $\mathbf{N}$ are the splitting polytope and the Newton polyhedron associated to $f$, then $\{|\mathbf{s}|: \mathbf{s} \in \mathbf{P} \backslash \mathbf{0}\}=\left\{\lambda>0: \frac{1}{\lambda} \cdot \mathbf{1}_{m} \in \mathbf{N}\right\}$. In particular,

$$
\max \{|\mathbf{s}|: \mathbf{s} \in \mathbf{P}\}=\max \left\{\lambda>0: \frac{1}{\lambda} \cdot \mathbf{1}_{m} \in \mathbf{N}\right\}
$$


Proof. Fix $\mathbf{s} \in \mathbf{P} \backslash \mathbf{0}$. By definition of $\mathbf{P}$, we have that $\mathbf{E s}=\sum s_{i} \cdot \mathbf{a}_{i} \preccurlyeq$ $\mathbf{1}_{m}$, and dividing this through by $|\mathbf{s}| \neq 0$, we see that $\sum \frac{s_{i}}{|\mathbf{s}|} \cdot \mathbf{a}_{i} \preccurlyeq \frac{1}{|\mathbf{s}|} \cdot \mathbf{1}_{m}$. Consequently, there exists $\boldsymbol{\delta} \in \mathbb{R}^{m}$ with $\boldsymbol{\delta} \succcurlyeq \mathbf{0}$ such that $\frac{1}{|\mathbf{s}|} \cdot \mathbf{1}_{m}=\sum \frac{s_{i}}{|\mathbf{s}|}$. $\mathbf{a}_{i}+\boldsymbol{\delta}$, and as $\sum \frac{s_{i}}{|\mathbf{s}|}=1$, this shows that $\frac{1}{|\mathbf{s}|} \cdot \mathbf{1}_{m} \in \mathbf{N}$.

Next, fix $\lambda \neq 0$ such that $\frac{1}{\lambda} \cdot \mathbf{1}_{m} \in \mathbf{N}$. By definition, there exist nonnegative real numbers $\sigma_{1}, \ldots, \sigma_{n}$ with $\sum \sigma_{i}=1$, and a vector $\boldsymbol{\delta} \succcurlyeq 0$ such that $\frac{1}{\lambda} \cdot \mathbf{1}_{m}=\sum \sigma_{i} \cdot \mathbf{a}_{i}+\boldsymbol{\delta}$, so that $\frac{1}{\lambda} \cdot \mathbf{1}_{m} \succcurlyeq \sum \sigma_{i} \cdot \mathbf{a}_{i}$. Multiplying through by $\lambda$ shows that $\mathbf{1}_{m} \succcurlyeq \sum\left(\lambda \sigma_{i}\right) \cdot \mathbf{a}_{i}$, and it follows that the vector $\mathbf{s}:=$ $\left(\lambda \sigma_{1}, \ldots, \lambda \sigma_{n}\right)$ is contained in $\mathbf{P}$, and has coordinate sum $\lambda$.

LEMMA 32. Suppose that $\mathbf{N}$ is in diagonal position, so that the ray generated by $\mathbf{1}_{m}$ intersects some bounded face of $\mathbf{N}$. If $\mathbf{F}$ is such a bounded face of $\mathbf{N}$, then

$$
\mathbf{P}_{\text {max }}=\left\{\mathbf{s} \in \mathbb{R}_{\geqslant 0}^{m}: \mathbf{E s}=\mathbf{1}_{m} \text { and } s_{i}=0 \text { whenever } \mathbf{a}_{i} \notin \mathbf{F}\right\} .
$$

Proof. By Lemma $28, \mathbf{F}=\mathrm{H}_{\beta}^{L} \cap \mathbf{N}$, where $\beta \in \mathbb{Q}_{>0}$, and $L \in \mathbb{Q}\left[z_{1}, \ldots z_{m}\right]$ is a positive linear combination of the variables. Moreover, by replacing $L$ with $\frac{1}{\beta} \cdot L$, we may, and will, assume that $\beta=1$. Given $\mathbf{s} \in \mathbf{P}_{\max }$, Lemma 31 implies that $(1 /|\mathbf{s}|) \cdot \mathbf{1}_{m}$, which lies on the ray generated by $\mathbf{1}_{m}$, is also contained in the boundary of $\mathbf{N}$, and hence lies in $\mathbf{F}$. Consequently,

$$
\frac{1}{|\mathbf{s}|} \cdot L\left(\mathbf{1}_{m}\right)=L\left(\frac{1}{|\mathbf{s}|} \cdot \mathbf{1}_{m}\right)=1 \text { for every } \mathbf{s} \in \mathbf{P}_{\max }
$$

Restated, $L\left(\mathbf{1}_{m}\right)=|\mathbf{s}|$ for every $\mathbf{s} \in \mathbf{P}_{\max }$, which shows that

$$
\mathbf{P}_{\max }=\left\{\mathbf{s} \in \mathbb{R}_{\geqslant 0}^{m}: \mathbf{E s} \preccurlyeq \mathbf{1}_{m} \text { and }|\mathbf{s}|=L\left(\mathbf{1}_{m}\right)\right\} .
$$

With this observation in hand, we begin our proof. Set

$$
\mathbf{Q}=\left\{\mathbf{s} \in \mathbb{R}_{\geqslant 0}^{m}: \mathbf{E s}=\mathbf{1}_{m} \text { and } s_{i}=0 \text { whenever } \mathbf{a}_{i} \notin \mathbf{F}\right\} .
$$

To complete the proof, we must show that $\mathbf{P}_{\max }=\mathbf{Q}$. Toward this end, choose $\mathbf{s} \in \mathbf{P}_{\max }$. As $L$ has positive coefficients, we may apply it to the bound $\mathbf{E s} \preccurlyeq \mathbf{1}_{m}$ to obtain

$$
|\mathbf{s}|=\sum_{i=1}^{n} s_{i} \leqslant \sum_{i=1}^{n} s_{i} \cdot L\left(\mathbf{a}_{i}\right)=L(\mathbf{E s}) \leqslant L\left(\mathbf{1}_{m}\right),
$$

where we have also used that $1 \leqslant L\left(\mathbf{a}_{i}\right)$ for all $1 \leqslant i \leqslant n$, by our choice of $L$. Furthermore, (4.2) shows that all of these inequalities must be, 
in fact, equalities. If the inequality $\mathbf{E s} \preccurlyeq \mathbf{1}_{m}$ were strict in some component, then the positivity of $L$ would imply that $L(\mathbf{E s})<L\left(\mathbf{1}_{m}\right)$, which is impossible by our previous observation. On the other hand, if $s_{k}$ were nonzero (and, hence, positive) for some index $k$ with $\mathbf{a}_{k} \notin \mathbf{F}$, then we would have that $1<L\left(\mathbf{a}_{k}\right)$, and, hence, than $s_{k}<s_{k} \cdot L\left(\mathbf{a}_{k}\right)$, which would then force the leftmost inequality above to be strict, which is also impossible. In summary, we have just shown that $\mathbf{s} \in \mathbf{Q}$.

Next, fix an element $\mathbf{s} \in \mathbf{Q}$, so that $\mathbf{1}_{m}=\mathbf{E s}=\sum_{\mathbf{a}_{i} \in \mathbf{F}} s_{i} \cdot \mathbf{a}_{i}$. By our choice of $L$, we have that $L\left(\mathbf{a}_{i}\right)=1$ whenever $\mathbf{a}_{i} \in \mathbf{F}$, and so applying $L$ to this identity shows that $L\left(\mathbf{1}_{m}\right)=\sum_{\mathbf{a}_{i} \in \mathbf{F}} s_{i}=|\mathbf{s}|$, which shows that $\mathbf{s} \in \mathbf{P}_{\max }$.

REMARK 33. (Constructing an element of $\mathbf{P}_{\max }$ ) It follows immediately from the definition that $\mathbf{P}_{\max }$ is nonempty. On the other hand, if $\mathbf{N}$ is in diagonal position, then one may also see this from the description given in the preceding lemma. Suppose $\lambda>0$ is such that $(1 / \lambda) \cdot \mathbf{1}_{m}$ lies in a bounded face $\mathbf{F}$ of Newton (such a $\lambda$ exists, by the assumption that $\mathbf{N}$ is in diagonal position), then because $\mathbf{F}$ is bounded, it is the convex hull of the set $\left\{\mathbf{a}_{1}, \ldots, \mathbf{a}_{n}\right\} \cap \mathbf{F}$, and so we may write both $(1 / \lambda) \mathbf{1}_{m}$ and $\mathbf{1}_{m}$ as a nonnegative combination of the elements of $\left\{\mathbf{a}_{1}, \ldots \mathbf{a}_{n}\right\} \cap \mathbf{F}$. The coefficients in this latter linear combination can be used to produce an element $\mathbf{s}$ described in the statement of Lemma 32.

The condition that ${ }^{\#} \mathbf{P}_{\max }=1$ appears multiple times in this article, and below we give an alternate description of this condition in terms of the Newton polyhedron $\mathbf{N}$ whenever $\mathbf{N}$ is in diagonal position.

REMARK 34. (A simple characterization of when ${ }^{\#} \mathbf{P}_{\max }=1$ ) Suppose, once more, that $\mathbf{N}$ is in diagonal position, and let $\mathbf{F}$ be the minimal bounded face of $\mathbf{N}$ that intersects the ray generated by $\mathbf{1}_{m}$. We claim that

$$
{ }^{\#} \mathbf{P}_{\max }=1 \Longleftrightarrow\left\{\mathbf{a}_{1}, \ldots, \mathbf{a}_{n}\right\} \cap \mathbf{F} \text { is a linearly independent subset of } \mathbb{R}^{m} \text {. }
$$

To see why this is the case, first suppose that $\left\{\mathbf{a}_{1}, \ldots, \mathbf{a}_{n}\right\} \cap \mathbf{F}$ is a linearly independent set. In this case, if $\mathbf{s}$ and $\mathbf{t}$ are in $\mathbf{P}_{\max }$, then Lemma 32 shows that the $k$ th entries of $\mathbf{s}$ and $\mathbf{t}$ are zero whenever $\mathbf{a}_{k} \notin \mathbf{F}$, and that

$$
\sum_{\mathbf{a}_{i} \in \mathbf{F}} t_{i} \cdot \mathbf{a}_{i}=\mathbf{E t}=\mathbf{1}_{m}=\mathbf{E s}=\sum_{\mathbf{a}_{i} \in \mathbf{F}} s_{i} \cdot \mathbf{a}_{i} .
$$

The linear independence of these vectors then shows that $s_{i}=t_{i}$ for every $i$ with $\mathbf{a}_{i} \in \mathbf{F}$, and so $\mathbf{s}=\mathbf{t}$. We conclude that ${ }^{\#} \mathbf{P}_{\max }=1$. 
Next, instead suppose that $\left\{\mathbf{a}_{1}, \ldots, \mathbf{a}_{n}\right\} \cap \mathbf{F}$ is not a linearly independent set, and fix coefficients so that $\sum_{\mathbf{a}_{i} \in \mathbf{F}} \delta_{i} \cdot \mathbf{a}_{i}=0$. As in Remark 33, we may write $\mathbf{1}_{m}$ as a nonnegative rational combination of the vectors in $\left\{\mathbf{a}_{1}, \ldots, \mathbf{a}_{n}\right\} \cap \mathbf{F}$, and the minimality of $\mathbf{F}$ guarantees that there exists such a linear combination with positive coefficients. Thus, we may fix positive rational coefficients $\gamma_{i}$ such that $\mathbf{1}_{m}=\sum_{\mathbf{a}_{i} \in \mathbf{F}} \gamma_{i} \cdot \mathbf{a}_{i}$, and this positivity implies that $\gamma_{i}+\varepsilon \cdot \delta_{i}>0$ for every $i$ with $\mathbf{a}_{i} \in \mathbf{F}$ whenever $\varepsilon>0$ is sufficiently small. Fix such an $\varepsilon$, and define an element $\mathbf{s} \in \mathbb{R}_{\geqslant 0}^{m}$ as follows: $s_{i}=0$ if $\mathbf{a}_{i} \notin \mathbf{F}$, and $s_{i}=\gamma_{i}+\varepsilon \cdot \delta_{i}$ otherwise. It follows that $s_{i}>0$ if and only if $\mathbf{a}_{i} \in \mathbf{F}$, and that

$$
\mathbf{E s}=\sum_{\mathbf{a}_{i} \in \mathbf{F}} s_{i} \cdot \mathbf{a}_{i}=\sum_{\mathbf{a}_{i} \in \mathbf{F}} \gamma_{i} \cdot \mathbf{a}_{i}+\varepsilon \cdot \sum_{\mathbf{a}_{i} \in \mathbf{F}} \delta_{i} \cdot \mathbf{a}_{i}=\sum_{\mathbf{a}_{i} \in \mathbf{F}} \gamma_{i} \cdot \mathbf{a}_{i}=\mathbf{1}_{m}
$$

This computation and Lemma 32 show that $\mathbf{s} \in \mathbf{P}_{\max }$, and as this holds for infinitely many values of $\varepsilon$, we have that ${ }^{\#} \mathbf{P}_{\max }>1$.

REMARK 35. (A comparison with another nondegeneracy condition on Newton polyhedra) Given a face $\sigma$ of $\mathbf{N}$, let $f_{\sigma}$ denote the polynomial obtained by summing only the terms of $f$ whose supporting monomials lie in $\sigma$. Recall that $f$ is said to be nondegenerate with respect to $\mathbf{N}$ whenever the system of equations $\partial f_{\sigma} / \partial x_{1}=\cdots=\partial f_{\sigma} / \partial x_{m}=0$ has no solution in $\left(\mathbb{L}^{*}\right)^{m}$ for every face $\sigma$ of $\mathbf{N}$. It is a general principle that polynomials that are nondegenerate with respect to their Newton polyhedra often satisfy certain desirable conditions (for a particularly relevant instance of this, see [How01b]).

Note that if $f \in \mathbb{L}\left[x_{1}, \ldots, x_{m}\right]$ is a general polynomial of degree $d$, then $f$ is nondegenerate with respect to $\mathbf{N}$. On the other hand, it is apparent that for such an $f$, the Newton polyhedron $\mathbf{N}$ is in diagonal position: the ray generated by $\mathbf{1}_{m}$ intersects $\mathbf{N}$ in the bounded face $\sigma=\left\{\mathbf{s} \in \mathbb{R}^{m}:|\mathbf{s}|=d\right\} \cap \mathbf{N}$ of $\mathbf{N}$. According to Remark 34, we then have that ${ }^{*} \mathbf{P}_{\max }=1$ if and only if the supporting monomials of $f$ lying on this face are linearly independent, which is impossible for dimension reasons. Thus, ${ }^{\#} \mathbf{P}_{\max }>1$.

As illustrated by this example, the condition that $f$ be nondegenerate with respect to $\mathbf{N}$ can be quite different from the condition that ${ }^{\#} \mathbf{P}_{\max }=1$. Indeed, as seen above, the latter condition may depend on the coefficients in $f$, while the former only depends on the supporting monomials of $f$. 


\section{$\S 5$. Polyhedra and F-pure thresholds}

Proposition 36. Fix a field $\mathbb{L}$ of characteristic $p>0$, and consider a polynomial $f$ in $\mathbb{L}\left[x_{1}, \ldots, x_{m}\right]$ vanishing at the origin. If $\mathbf{P}$ is the splitting polytope associated to $f$, and $\mathfrak{a}_{f}$ is the term ideal of $f$, then $\operatorname{fpt}\left(\mathfrak{a}_{f}\right)=$ $\max \{|\mathbf{s}|: \mathbf{s} \in \mathbf{P}\}=\max \left\{\lambda \neq 0: \frac{1}{\lambda} \cdot \mathbf{1}_{m} \in \mathbf{N}\right\}$.

Proof. Fix distinct, nonzero vectors in $\mathbb{N}^{m}$ such that $\operatorname{Supp}(f)=$ $\left\{\mathbf{a}_{1}, \ldots, \mathbf{a}_{n}\right\}$. By Lemma 31, it suffices to establish the first equality. By definition, $\mathfrak{a}:=\mathfrak{a}_{f}=\left(x^{\mathfrak{a}_{1}}, \ldots, x^{\mathbf{a}_{n}}\right)$, and hence the ideal $\mathfrak{a}^{N}$ is generated by monomials of the form $x^{k_{1} \mathbf{a}_{1}+\cdots+k_{n} \mathbf{a}_{n}}=x^{\mathbf{E k}}$ with $|\mathbf{k}|=k_{1}+\cdots+k_{n}=N$. As $\mathfrak{a}^{\nu_{\mathfrak{a}}\left(p^{e}\right)} \notin \mathfrak{m}^{\left[p^{e}\right]}$, there exists $\mathbf{k} \in \mathbb{N}^{n}$ with $|\mathbf{k}|=\nu_{\mathfrak{a}}\left(p^{e}\right)$ such that $\boldsymbol{x}^{\mathbf{E k}} \notin$ $\mathfrak{m}^{\left[p^{e}\right]}$. By definition, $p^{-e} \cdot \mathbf{k} \in \mathbf{P}$, and consequently $p^{-e} \cdot \nu_{\mathfrak{a}}\left(p^{e}\right)=p^{-e} \cdot|\mathbf{k}|=$ $\left|p^{-e} \cdot \mathbf{k}\right|$, which by definition is bounded above by $\max \{|\mathbf{s}|: \mathbf{s} \in \mathbf{P}\}$. Taking $e \rightarrow \infty$ then shows that $\mathbf{f p t}(\mathfrak{a}) \leqslant \max \{|\mathbf{s}|: \mathbf{s} \in \mathbf{P}\}$. Next, choose $\boldsymbol{\eta} \in \mathbf{P}_{\max }$. As $\langle\boldsymbol{\eta}\rangle_{e} \prec \boldsymbol{\eta}$, we have that $\mathbf{E}\langle\boldsymbol{\eta}\rangle_{e} \prec \mathbf{E} \boldsymbol{\eta} \preccurlyeq \mathbf{1}_{m}$, and so $x^{p^{e} \mathbf{E}\langle\boldsymbol{\eta}\rangle_{e}}$ is contained in $\mathfrak{a}^{p^{e}}\left|\langle\boldsymbol{\eta}\rangle_{e}\right|$ but not $\mathfrak{m}^{\left[p^{e}\right]}$. It follows that $p^{-e} \cdot \nu_{\mathfrak{a}}\left(p^{e}\right) \geqslant\left|\langle\boldsymbol{\eta}\rangle_{e}\right|$, and letting $e \rightarrow \infty$ shows that $\boldsymbol{f p t}(\mathfrak{a}) \geqslant|\boldsymbol{\eta}|=\max \{|\mathbf{s}|: \mathbf{s} \in \mathbf{P}\}$.

COROllary 37. If $\mathbb{L}$ is a field of characteristic $p>0$, and $f$ in $\mathbb{L}\left[x_{1}, \ldots, x_{m}\right]$ is a polynomial vanishing at the origin, then the following are equivalent.

(1) There exists a maximal point $\boldsymbol{\eta} \in \mathbf{P}_{\max }$ with $\left(p^{e}-1\right) \cdot \boldsymbol{\eta} \in \mathbb{N}^{n}$.

(2) $\left(p^{e}-1\right) \cdot \mathbf{f} \mathbf{p t}\left(\mathfrak{a}_{f}\right) \in \mathbb{N}$ and $\mathfrak{a}_{f}^{\left(p^{e}-1\right) \cdot \mathbf{f p t}\left(\mathfrak{a}_{f}\right)} \notin \mathfrak{m}^{\left[p^{e}\right]}$.

Furthermore, if either condition holds and $\mathbf{N}$ is in diagonal position, then

$$
\mathfrak{a}_{f}^{\left(p^{e}-1\right) \cdot \operatorname{fpt}\left(\mathfrak{a}_{f}\right)} \equiv\left(x_{1}^{p^{e}-1} \cdots x_{m}^{p^{e}-1}\right) \bmod \mathfrak{m}^{\left[p^{e}\right]} .
$$

Proof. Set $\operatorname{Supp}(f)=\left\{\mathbf{a}_{1}, \ldots, \mathbf{a}_{n}\right\}$, and $\mathfrak{a}=\mathfrak{a}_{f}$. By definition, $\mathfrak{a}^{N}$ is generated by the monomials $\boldsymbol{x}^{\text {Ek }}$ with $|\mathbf{k}|=N$. If $\boldsymbol{\eta} \in \mathbf{P}_{\max }$ and $\left(p^{e}-1\right)$. $\boldsymbol{\eta} \in \mathbb{N}^{n}$, then it follows from Proposition 36 that $\left(p^{e}-1\right) \cdot \mathbf{f p t}(\mathfrak{a})=\left(p^{e}-\right.$

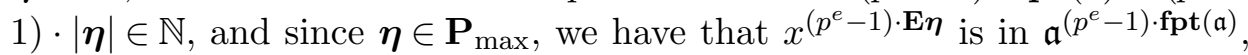
but not in $\mathfrak{m}^{\left[p^{e}\right]}$. Conversely, if $\left(p^{e}-1\right) \cdot \mathbf{f p t}(\mathfrak{a}) \in \mathbb{N}$, and $\mathfrak{a}^{\left(p^{e}-1\right) \cdot \operatorname{fpt}(\mathfrak{a})} \nsubseteq \mathfrak{m}^{\left[p^{e}\right]}$, there exists $\mathbf{k} \in \mathbb{N}^{n}$ such that $|\mathbf{k}|=\left(p^{e}-1\right) \cdot \mathbf{f p t}(\mathfrak{a})$ and $\boldsymbol{x}^{\mathbf{E k}} \notin \mathfrak{m}^{\left[p^{e}\right]}$, so that $\frac{1}{p^{e}-1} \cdot \mathbf{k} \in \mathbf{P}_{\text {max }}$. Having proven the equivalence, it remains to establish the last assertion, which we do now. We have just seen that the monomials in $\mathfrak{a}^{\left(p^{e}-1\right) \cdot \mathbf{f p t}(\mathfrak{a})}$ not contained in $\mathfrak{m}^{\left[p^{e}\right]}$ are of the form $\boldsymbol{x}^{\mathbf{E k}}$ for some index $\mathbf{k}$ satisfying $\frac{1}{p^{e}-1} \cdot \mathbf{k} \in \mathbf{P}_{\max }$. By Lemma $32, \mathbf{E k}=\left(p^{e}-1\right) \cdot \mathbf{1}_{m}$, and it follows that the only monomial in $\mathfrak{a}^{\left(p^{e}-1\right) \cdot \mathfrak{f p t}(\mathfrak{a})}$ not in $\mathfrak{m}^{\left[p^{e}\right]}$ is $x^{\left(p^{e}-1\right) \cdot \mathbf{1}_{m}}$. 
Proposition 38. Fix a field $\mathbb{L}$ of characteristic $p$, and a polynomial $f \in \mathbb{L}\left[x_{1}, \ldots, x_{m}\right]$ vanishing at the origin. We fix distinct, nonzero vectors $\mathbf{a}_{1}, \ldots, \mathbf{a}_{n}$ in $\mathbb{N}^{m}$, and nonzero elements $u_{1}, \ldots, u_{n}$ in $\mathbb{L}$, so that $f=$ $\sum_{i=1}^{n} u_{i} \cdot x^{\mathbf{a}_{i}}$. If $\mathbf{N}$ is in diagonal position, and if $\left(p^{e}-1\right) \cdot \boldsymbol{\eta} \in \mathbb{N}^{n}$ for some $\boldsymbol{\eta} \in \mathbf{P}_{\max }$ and $e \geqslant 1$, then there exists a nonzero polynomial $\Theta \in$ $\mathbb{Z}\left[w_{1}, \ldots, w_{n}\right]$ satisfying the following conditions.

(1) $f^{\left(p^{e}-1\right) \cdot \operatorname{fpt}\left(\mathfrak{a}_{f}\right)} \equiv \Theta\left(u_{1}, \ldots, u_{r}\right) \cdot x_{1}^{p^{e}-1} \cdots x_{m}^{p^{e}-1} \bmod \mathfrak{m}^{\left[p^{e}\right]}$.

(2) If $e=1$ and $\mathbf{f p t}\left(\mathfrak{a}_{f}\right) \leqslant 1$, then $\Theta$ has nonzero image in $\mathbb{F}_{p}\left[w_{1}, \ldots, w_{n}\right]$.

Proof. Set $\mathfrak{a}=\mathfrak{a}_{f}$. By definition, $f^{\left(p^{e}-1\right) \cdot \mathbf{f p t}(\mathfrak{a})} \in \mathfrak{a}^{\left(p^{e}-1\right) \cdot \mathbf{f p t}(\mathfrak{a})}$, and Corollary 37 then implies that $f^{\left(p^{e}-1\right) \cdot \operatorname{fpt}(\mathfrak{a})}$ is a multiple of $x^{\left(p^{e}-1\right) \cdot \mathbf{1}_{m}} \bmod$ ulo $\mathfrak{m}^{\left[p^{e}\right]}$. Moreover, the multinomial theorem identifies this multiple as $\Theta\left(u_{1}, \ldots, u_{r}\right) \cdot x^{\left(p^{e}-1\right) \cdot \mathbf{1}_{m}} \bmod \mathfrak{m}^{\left[p^{e}\right]}$, where

$$
\Theta\left(w_{1}, \ldots, w_{n}\right):=\sum\left(\begin{array}{c}
\left(p^{e}-1\right) \cdot \mathbf{f p t}(\mathfrak{a}) \\
k_{1}, \ldots, k_{n}
\end{array}\right) w_{1}^{k_{1}} \cdots w_{n}^{k_{n}} \in \mathbb{Z}\left[w_{1}, \ldots, w_{r}\right],
$$

and the sum in (5.1) extends over all $\mathbf{k}=\left(k_{1}, \ldots, k_{n}\right) \in \mathbb{N}^{n}$ such that $|\mathbf{k}|=$ $\left(p^{e}-1\right) \cdot \mathbf{f p t}(\mathfrak{a})$. (Note that, for such an index, we have that $\frac{1}{p^{e}-1} \cdot \mathbf{k}$ is in $\mathbf{P}_{\max }$, and, hence, Lemma 32 implies that $\mathbf{E k}=\left(p^{e}-1\right) \cdot \mathbf{1}_{m}$, making this condition redundant.) By assumption, there exists $\boldsymbol{\eta} \in \mathbf{P}_{\max }$ with $\left(p^{e}-\right.$ 1) $\cdot \boldsymbol{\eta} \in \mathbb{N}^{n}$; as this index corresponds to a nonzero summand in (5.1), we conclude that $\Theta$ is a nonzero polynomial over $\mathbb{Z}$. For the last point, note that if $\mathbf{f p t}(\mathfrak{a}) \leqslant 1$, then $(p-1) \cdot \mathbf{f p t}(\mathfrak{a}) \leqslant p-1$, and hence the multinomial coefficients in (5.1) are nonzero modulo $p$.

Corollary 39. Fix a field $\mathbb{L}$ of characteristic $p$, and a polynomial $f \in \mathbb{L}\left[x_{1}, \ldots, x_{m}\right]$ vanishing at the origin. We fix distinct, nonzero vectors $\mathbf{a}_{1}, \ldots, \mathbf{a}_{n}$ in $\mathbb{N}^{m}$, and nonzero elements $u_{1}, \ldots, u_{n}$ in $\mathbb{L}$, so that $f=$ $\sum_{i=1}^{n} u_{i} \cdot x^{\mathbf{a}_{i}}$. If $\mathbf{N}$ is in diagonal position, and if $\mathbf{P}$ contains a unique maximal point $\boldsymbol{\eta}$ such that $\left(p^{e}-1\right) \cdot \boldsymbol{\eta} \in \mathbb{N}^{n}$ for some $e \geqslant 1$, then

$$
f^{\left(p^{e}-1\right) \cdot \mathbf{f p t}\left(\mathfrak{a}_{f}\right)} \equiv\left(\begin{array}{c}
\left(p^{e}-1\right) \cdot \mathbf{f} \mathbf{p t}\left(\mathfrak{a}_{f}\right) \\
\left(p^{e}-1\right) \cdot \boldsymbol{\eta}
\end{array}\right) u^{\left(p^{e}-1\right) \boldsymbol{\eta}} \cdot x^{\left(p^{e}-1\right) \cdot \mathbf{1}_{m}} \bmod \mathfrak{m}^{\left[p^{e}\right]} .
$$

Proof. As $\mathbf{P}_{\max }=\{\boldsymbol{\eta}\}$, (5.1) implies that the only summand appearing in $\Theta$ corresponds to $\mathbf{k}=\left(p^{e}-1\right) \cdot \boldsymbol{\eta}$, and the claim then follows from Proposition 38.

Proposition 40. Fix a field $\mathbb{L}$ of characteristic $p$, and a polynomial $f \in \mathbb{L}\left[x_{1}, \ldots, x_{m}\right]$ vanishing at the origin. We fix distinct, nonzero vectors 
$\mathbf{a}_{1}, \ldots, \mathbf{a}_{n}$ in $\mathbb{N}^{m}$, and nonzero elements $u_{1}, \ldots, u_{n}$ in $\mathbb{L}$, so that $f=$ $\sum_{i=1}^{n} u_{i} \cdot x^{\mathbf{a}_{i}}$. If $\mathbf{f p t}\left(\mathfrak{a}_{f}\right) \leqslant 1, \mathbf{N}$ is in diagonal position, and $(p-1) \cdot \boldsymbol{\eta} \in \mathbb{N}^{n}$ for some $\boldsymbol{\eta} \in \mathbf{P}_{\max }$, then there exists a nonempty closed set $Z \subseteq \mathbb{A}_{\mathbb{L}}^{n}$ such that $\mathbf{f p t}(f)=\mathbf{f p t}\left(\mathfrak{a}_{f}\right)$ whenever $\left(u_{1}, \ldots, u_{n}\right) \notin Z$.

Proof. Let $\Theta \in \mathbb{Z}\left[w_{1}, \ldots, w_{n}\right]$ be as in Proposition 38. The assumption that $\mathbf{f p t}\left(\mathfrak{a}_{f}\right) \leqslant 1$ implies that $\Theta$ is nonzero modulo $p$, and hence defines a nonempty closed set $Z$ in $\mathbb{A}_{\mathbb{R}}^{n}$. Moreover, Proposition 38 shows that, if

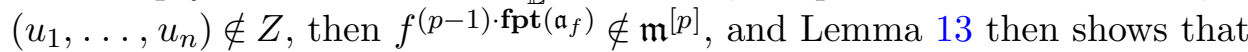
$\mathbf{f p t}(f) \geqslant \mathbf{f p t}\left(\mathfrak{a}_{f}\right)$. As the opposite inequality always holds, we conclude that $\mathbf{f p t}(f)=\mathbf{f p t}\left(\mathfrak{a}_{f}\right)$ whenever $\left(u_{1}, \ldots, u_{n}\right) \notin Z$.

Remark 41. The condition that $(p-1) \cdot \boldsymbol{\eta} \in \mathbb{N}^{n}$ in Proposition 40 is necessary. Indeed, if $f=u_{1} x_{d}^{d}+\cdots+u_{d} x_{d}^{d}$ is a degree $d$ Fermat hypersurface, we have seen in Example 20 that $\boldsymbol{\eta}=\left(\frac{1}{d}, \ldots, \frac{1}{d}\right)$ is the unique maximal point of $\mathbf{P}$, so that $\mathbf{f p t}\left(\mathfrak{a}_{f}\right)=\frac{1}{d}+\cdots+\frac{1}{d}=1$ by Proposition 36 . However, it is shown in [Her15] that, if $(p-1) \cdot \frac{1}{d} \notin \mathbb{N}$, then $\mathbf{f p t}(f)<\mathbf{f p t}\left(\mathfrak{a}_{f}\right)$ for every choice of coefficients $\left(u_{1}, \ldots, u_{n}\right)$.

ThEOREM 42. Fix a field $\mathbb{L}$ of characteristic $p$, and a polynomial $f \in \mathbb{L}\left[x_{1}, \ldots, x_{m}\right]$ vanishing at the origin. Suppose that the associated splitting polytope $\mathbf{P}$ contains a unique maximal point $\boldsymbol{\eta}$, and let $L=$ $\inf \left\{e \geqslant 0: \eta_{1}{ }^{(e+1)}+\cdots+\eta_{n}{ }^{(e+1)} \geqslant p\right\}$, where $\eta_{i}^{(e)}$ is the eth digit of $\eta_{i}$.

(1) If $L=\infty$, then $\mathbf{f p t}(f)=\mathbf{f p t}\left(\mathfrak{a}_{f}\right)$. The converse holds if $\mathbf{N}$ is in diagonal position and $p$ does not divide any of the denominators of $\boldsymbol{\eta}$.

(2) If $L<\infty$, then $\mathbf{f p t}(f) \geqslant\left\langle\eta_{1}\right\rangle_{L}+\cdots+\left\langle\eta_{n}\right\rangle_{L}+\frac{1}{p^{L}}$.

Proof. We fix distinct, nonzero vectors $\mathbf{a}_{1}, \ldots, \mathbf{a}_{n}$ in $\mathbb{N}^{m}$, and nonzero elements $u_{1}, \ldots, u_{n}$ in $\mathbb{L}$, so that $f=\sum_{i=1}^{n} u_{i} \cdot x^{\mathbf{a}_{i}}$. We first prove (1), and thus assume that $L=\infty$ (i.e., that entries of $\boldsymbol{\eta}$ add without carrying). Corollary 25 gives that, after gathering terms,

$$
\left(\begin{array}{c}
p^{e}\left|\langle\boldsymbol{\eta}\rangle_{e}\right| \\
p^{e}\langle\boldsymbol{\eta}\rangle_{e}
\end{array}\right) u^{p^{e}\langle\boldsymbol{\eta}\rangle_{e}} x^{p^{e} \mathbf{E}\langle\boldsymbol{\eta}\rangle_{e}}
$$

appears as a summand of $f^{p^{e}\left|\langle\boldsymbol{\eta}\rangle_{e}\right|}$. By definition, each $u_{i} \in \mathbb{L}^{*}$, so $u^{p^{e}}\langle\boldsymbol{\eta}\rangle_{e} \neq$ 0 . Moreover, as the entries of $\boldsymbol{\eta}$ add without carrying, it follows from Corollary 7 that $\left(\begin{array}{c}p^{e}\left|\langle\boldsymbol{\eta}\rangle_{e}\right| \\ p^{e}\langle\boldsymbol{\eta}\rangle_{e}\end{array}\right) \neq 0 \bmod p$ for every $e \geqslant 1$. Finally, $\langle\boldsymbol{\eta}\rangle_{e} \preccurlyeq \boldsymbol{\eta}$, and so every entry of $p^{e} \mathbf{E}\langle\boldsymbol{\eta}\rangle_{e}$ is less than or equal to $p^{e}-1$. We see then that the monomial in (5.2) is in $\operatorname{Supp}\left(f^{p^{e}\left|\langle\boldsymbol{\eta}\rangle_{e}\right|}\right)$, but not in $\mathfrak{m}^{\left[p^{e}\right]}$. 
Thus, $f^{p^{e}}\left|\langle\boldsymbol{\eta}\rangle_{e}\right| \notin \mathfrak{m}^{\left[p^{e}\right]}$, and so $\frac{\nu_{f}\left(p^{e}\right)}{p^{e}} \geqslant\left|\langle\boldsymbol{\eta}\rangle_{e}\right|$. Letting $e \rightarrow \infty$ shows that $\boldsymbol{f p t}(f) \geqslant|\boldsymbol{\eta}|=\boldsymbol{f p t}\left(\mathfrak{a}_{f}\right)$, where the last inequality follows from Proposition 36. As the opposite inequality always holds, we have just shown that $\mathbf{f p t}(f)=\mathbf{f p t}\left(\mathfrak{a}_{f}\right)$ if $L=\infty$, and it remains to show that the converse holds if $\mathbf{N}$ is in diagonal position, and $p$ does not divide any denominator in $\boldsymbol{\eta}$, which we do now. Suppose that $\mathbf{f p t}(f)=\mathbf{f p t}\left(\mathfrak{a}_{f}\right)$. Our hypothesis on $p$ implies that $\left(p^{e}-1\right) \cdot \boldsymbol{\eta} \in \mathbb{N}$ for infinitely many $e$, and for such $e \geqslant 1$, Lemma 13 implies

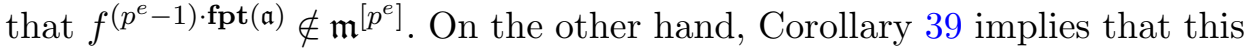
occurs if and only if

$$
\left(\begin{array}{c}
\left(p^{e}-1\right) \cdot \mathbf{f p t}\left(\mathfrak{a}_{f}\right) \\
\left(p^{e}-1\right) \cdot \boldsymbol{\eta}
\end{array}\right) \neq 0 \bmod p .
$$

Finally, Lemma 3 and Corollary 7 show that this multinomial coefficient is nonzero $\bmod p$ if and only if the entries of $\boldsymbol{\eta}$ add without carrying.

We now address the second point. If $L<\infty$, then $\eta_{1}{ }^{(L+1)}+\cdots+$ $\eta_{n}{ }^{(L+1)} \geqslant p$, by definition. Consequently, there exist (not necessarily unique) integers $\delta_{1}, \ldots, \delta_{n}$ such that

$$
\delta_{1}+\cdots+\delta_{n}=p-1 \text { and } 0 \leqslant \delta_{i} \leqslant \eta_{i}{ }^{(L+1)},
$$

with the second inequality in (5.3) being strict for at least one index. Without loss of generality, we assume that $\delta_{1}<\eta_{1}{ }^{(L+1)}$. For $e \geqslant 2$, set

$$
\boldsymbol{\lambda}=\langle\boldsymbol{\eta}\rangle_{L}+\left(\frac{\delta_{1}}{p^{L+1}}+\frac{p-1}{p^{L+2}}+\cdots+\frac{p-1}{p^{L+e}}, \frac{\delta_{2}}{p^{L+1}}, \ldots, \frac{\delta_{n}}{p^{L+1}}\right) .
$$

We summarize some important properties of $\boldsymbol{\lambda}$. By construction, $p^{L+e}$. $\boldsymbol{\lambda} \in \mathbb{N}^{n}$. Furthermore, the defining properties of the $\delta_{i}$ show that both $\boldsymbol{\lambda} \prec$ $\langle\boldsymbol{\eta}\rangle_{e}$ and

$$
|\boldsymbol{\lambda}|=\left|\langle\boldsymbol{\eta}\rangle_{L}\right|+\frac{p-1}{p^{L+1}}+\frac{p-1}{p^{L+2}}+\cdots+\frac{p-1}{p^{L+e}}=\left|\langle\boldsymbol{\eta}\rangle_{L}\right|+\frac{p^{e}-1}{p^{L+e}},
$$

while the definition of $L$, and Lemma 6 , imply that $\left(\begin{array}{c}p^{L+e}|\boldsymbol{\lambda}| \\ p^{L+e} \boldsymbol{\lambda}\end{array}\right) \neq 0$ modulo $p$. These properties imply that $\left(\begin{array}{c}p^{L+e}|\boldsymbol{\lambda}| \\ p^{L+e} \boldsymbol{\lambda}\end{array}\right) u^{p^{L+e} \boldsymbol{\lambda}} x^{p^{L+e} \mathbf{E} \boldsymbol{\lambda}}$ is a nonzero summand of $f^{p^{L+e}|\boldsymbol{\lambda}|}=f^{p^{L+e}\left|\langle\boldsymbol{\eta}\rangle_{L}\right|+p^{e}-1}$ that is not contained in $\mathfrak{m}^{\left[p^{L+e}\right]}$, so that $\frac{\nu_{f}\left(p^{L+e}\right)}{p^{L+e}} \geqslant\left|\langle\boldsymbol{\eta}\rangle_{L}\right|+\frac{p^{e}-1}{p^{L+e}}$, and the assertion follows by letting $e \rightarrow \infty$.

REMARK 43. The estimates given in Theorem 42 can be used to calculate $\mathbf{f p t}_{\mathfrak{m}}(f)$ in any characteristic whenever $f$ is either a diagonal or binomial hypersurface (see [Her15, Her14]). 


\section{§6. Log canonical singularities and (dense/open) F-pure type}

In this section, we prove our main results relating $F$-pure and log canonical thresholds. Although it is not needed in this article, we briefly recall the definition of the $\log$ canonical threshold. Given an ideal $\mathfrak{a} \subseteq$ $\mathbb{C}\left[x_{1}, \ldots, x_{m}\right]$, recall that a $\log$ resolution of $\mathfrak{a}$ consists of a map $\pi: X \rightarrow \mathbb{A}_{\mathbb{C}}^{m}$ with the following properties.

- $X$ is a smooth variety over $\mathbb{C}$.

- $\pi$ is a proper, birational map of algebraic varieties.

- $\mathfrak{a} \cdot \mathcal{O}_{X}$ is invertible, and hence of the form $\mathcal{O}_{X}(-D)$ for some effective divisor $D$.

- If $E_{\pi}$ is the exceptional divisor of $\pi$, then $E_{\pi}+D$ has simple normal crossing support.

Given any log resolution, we use $D_{\pi}^{\mathfrak{a}}$ to denote an effective divisor on $X$ such that $\mathfrak{a} \cdot \mathcal{O}_{X}=\mathcal{O}_{X}\left(-D_{\pi}^{\mathfrak{a}}\right)$, and we use $K_{\pi}$ to denote a relative canonical divisor of $\pi$ (which we may also choose to be effective). Finally, if $\mathbf{z}$ is any point in $\mathbb{A}_{\mathbb{C}}^{m}$, and $D=\sum a_{i} \cdot D_{i}$ is any $\mathbb{R}$-divisor on $X$ (here, the sum extends over all prime divisors on $X$, so that $a_{i}=0$ for almost all $i$ ), we say that $D$ is $\pi$-effective over $\mathbf{z}$ if the divisor $\sum_{\mathbf{z} \in \pi\left(D_{i}\right)} a_{i} \cdot D_{i}$ is effective.

Definition 44. Fix an ideal $\mathfrak{a} \subseteq \mathbb{C}\left[x_{1}, \ldots, x_{m}\right]$ with $\mathbf{0} \in \mathbb{V}(\mathfrak{a})$, and consider a $\log$ resolution $\pi: X \rightarrow \mathbb{A}_{\mathbb{C}}^{m}$ of $\mathfrak{a}$. The $\log$ canonical threshold of $\mathfrak{a}$ at the origin is defined as

$$
\begin{array}{r}
\operatorname{lct}(\mathfrak{a}):=\sup \left\{\lambda>0 \text { : the divisor } K_{\pi}-\lambda \cdot D_{\pi}^{\mathfrak{a}}\right. \\
\text { on } \left.X \text { is } \pi \text {-effective over } \mathbf{0} \in \mathbb{A}_{\mathbb{C}}^{m}\right\} .
\end{array}
$$

In the case that $\mathfrak{a}$ is a principal ideal generated by $f$, we write $\operatorname{lct}(f)$ instead of $\operatorname{lct}(\mathfrak{a})$.

The log canonical threshold turns out to not depend on the choice of $\log$ resolution. Moreover, $\operatorname{lct}(\mathfrak{a})$ is always a rational number, and the $\log$ canonical threshold of a principal ideal is always contained in $(0,1]$. Furthermore, if $f \in \mathfrak{a}$, then $\operatorname{lct}(f) \leqslant \operatorname{lct}(\mathfrak{a})$. For justification of these facts, and for more references to the theory of log canonical thresholds (and related topics), we refer the reader to the surveys [BL04, EM06, BFS13]. In what follows, we summarize all of the properties of log canonical thresholds that we need in this article. 
Proposition 45. Let $f \in \mathbb{C}\left[x_{1}, \ldots, x_{m}\right]$ be a polynomial vanishing at the origin, and let $\mathfrak{a}_{f}$ denote its term ideal, so that $\mathbf{0}$ is contained in both the vanishing set of $f$ and $\mathfrak{a}_{f}$.

(1) $\operatorname{lct}(f)$ and $\mathbf{l c t}\left(\mathfrak{a}_{f}\right)$ are rational numbers, with $\operatorname{lct}(f) \leqslant \min \left\{1, \operatorname{lct}\left(\mathfrak{a}_{f}\right)\right\}$.

(2) If $\mathbf{N}$ denotes the Newton polyhedron associated to $f$, then

$$
\operatorname{lct}\left(\mathfrak{a}_{f}\right)=\max \left\{\lambda>0: \frac{1}{\lambda} \cdot \mathbf{1}_{m} \in \mathbf{N}\right\}
$$

Proof. The first point follows from the preceding discussion, while the second appears in [How01a].

COROllary 46. If $\mathbb{L}$ is a field of characteristic $p>0$, and if $f \in$ $\mathbb{C}\left[x_{1}, \ldots, x_{m}\right]$ and $f_{p} \in \mathbb{L}\left[x_{1}, \ldots, x_{m}\right]$ are polynomials vanishing at the origin with $\operatorname{Supp}(f)=\operatorname{Supp}\left(f_{p}\right)$, then $\mathbf{f p t}\left(\mathfrak{a}_{f}\right)=\operatorname{lct}\left(\mathfrak{a}_{f_{p}}\right)$.

Proof. This result is a restatement of [HY03, Theorem 6.10(3)]. For a self-contained proof, compare the description of $\operatorname{lct}\left(\mathfrak{a}_{f}\right)$ given in Proposition 45 with the description of $\mathbf{f p t}\left(\mathfrak{a}_{f_{p}}\right)$ given in Proposition 36.

\subsection{On reduction to positive characteristic}

In this subsection, we discuss the process of reduction to positive characteristic. Many of the technical steps that follow depend on the following well-known lemma.

LEMma 47. Let $A$ be a finitely generated algebra over a domain $D \subseteq$ A. There exists a nonzero element $N \in D$ such that $D_{N} \subseteq A_{N}$ factors as $D_{N} \subseteq D_{N}\left[z_{1}, \ldots, z_{d}\right] \subseteq A_{N}$, where $z_{1}, \ldots, z_{d}$ are algebraically independent over $D_{N}$, and $D_{N}\left[z_{1}, \ldots, z_{d}\right] \subseteq A_{N}$ is finite.

Proof. If $L=$ Frac $D$, and $R$ is the localization of $A$ at the nonzero elements of $D$, then Lemma 47 can be obtained by applying the Noether normalization theorem to the inclusion $L \subseteq R$. The reader is referred to the author's thesis [Her11] for more details.

Corollary 48. If $A$ is a finitely generated $\mathbb{Z}$-algebra, then $A / \mu$ is a finite field for every maximal ideal $\mu \subseteq$ A. Furthermore, all but finitely many primes $p \in \mathbb{Z}$ are contained in a maximal ideal of $A$. 
Proof. This follows from Lemma 47, and we refer the reader to the author's thesis [Her11] for a detailed proof.

Suppose that $f \in \mathbb{C}\left[x_{1}, \ldots, x_{m}\right]$ is a polynomial vanishing at the origin, and let $A \subseteq \mathbb{C}$ be a finitely generated $\mathbb{Z}$-algebra containing the coefficients of $f$, so that $f \in A\left[x_{1}, \ldots, x_{m}\right]$. For such an algebra, it follows from Corollary 48 that $A / \mu$ is a finite field for every $\mu \in \operatorname{mSpec} A$, and all but finitely many primes appear in the set $\{\operatorname{char} A / \mu: \mu \in \operatorname{mSpec} A\}$. Let $f_{\mu}$ denote the image of $f$ in $(A / \mu)\left[x_{1}, \ldots, x_{m}\right]$. Furthermore, we may enlarge $A$ (say, by adjoining the inverses of the coefficients of $f$ ) so as to assume that $\operatorname{Supp}\left(f_{\mu}\right)=\operatorname{Supp}(f)$ for every $\mu \in \operatorname{mSpec} A$. We call $\left\{f_{\mu}: \mu \in \operatorname{mSpec} A\right\}$ a family of positive characteristic models of $f$.

Corollary 49. If $A$ is a finitely generated algebra over a domain $D \subseteq$ $A$, then the inverse image of a dense set under the induced map $\operatorname{Spec} A \stackrel{\bar{\pi}}{\rightarrow}$ Spec $D$ is also dense.

Proof. Let $\mathscr{D}$ be dense in Spec $D$. It suffices to show that $\operatorname{Spec} A_{f} \cap$ $\pi^{-1}(\mathscr{D})$ is nonempty for every nonzero $f \in A$. As $A$ is finitely generated over $D$, so is $A_{f}=A[T] /(1-T f)$. Consider a factorization $D_{N} \subseteq$ $D_{N}\left[z_{1}, \ldots, z_{d}\right] \subseteq A_{f N}$ as in Lemma 47. By the lying over theorem, Spec $A_{f N} \stackrel{\pi}{\rightarrow}$ Spec $D_{N}$ is surjective. As $\mathscr{D}$ is dense, $\mathscr{D} \cap \operatorname{Spec} D_{N}=\mathscr{D} \cap$ $\pi\left(\operatorname{Spec} A_{f N}\right)$ is nonempty. Consequently, Spec $A_{f N} \cap \pi^{-1}(\mathscr{D})$, and hence Spec $A_{f} \cap \pi^{-1}(\mathscr{D})$, is nonempty.

\subsection{Connections with $F$-pure thresholds}

Notation 50. Set $S=\mathbb{C}\left[x_{1}, \ldots, x_{m}\right]$. If $A$ is a finitely generated $\mathbb{Z}$ subalgebra of $\mathbb{C}$, we use $S_{A}$ to denote the subring $A\left[x_{1}, \ldots, x_{m}\right] \subseteq S$. Note that $\mathbb{C} \otimes_{A} S_{A}=S$. If $\mu$ is a maximal ideal of $A, S_{A}(\mu)$ denotes the polynomial ring $S_{A} \otimes_{A} A / \mu=S_{A} / \mu S_{A}=(A / \mu)\left[x_{1}, \ldots, x_{m}\right]$. By Corollary 48 , char $S_{A}(\mu)>0$. For $f \in S_{A}$, we use $f_{\mu}$ to denote the image of $f$ in $S_{A}(\mu)$. Finally, $\mathfrak{m}$ denotes the ideal generated by the variables $x_{1}, \ldots, x_{m}$ in the polynomial rings $S, S_{A}$, and $S_{A}(\mu)$.

It is an important fact that the $F$-pure (respectively, log canonical) threshold of a polynomial may also be defined in terms of its associated test ideals (respectively, multiplier ideals). Theorem 51 below was first observed in [MTW05, Theorem 3.4], and follows from deep theorems in [Smi00, HY03] relating test ideals and multiplier ideals. We refer the reader to the author's thesis [Her11] for a detailed proof. 
THEOREM 51. Let $f \in S$ be a polynomial vanishing at the origin. Then, for every finitely generated $\mathbb{Z}$-algebra $A \subseteq \mathbb{C}$ with $f \in S_{A}$, the following hold.

(1) There exists a dense open set $U \subseteq \operatorname{Spec} A$ such that $\operatorname{fpt}\left(f_{\mu}\right) \leqslant \operatorname{lct}(f)$ for every maximal ideal $\mu \in U$.

(2) For every $0<\lambda<\operatorname{lct}(f)$, there exists a dense open set $U_{\lambda} \subseteq \operatorname{Spec} A$ such that $\lambda \leqslant \operatorname{fpt}\left(f_{\mu}\right) \leqslant \operatorname{lct}(f)$ for every maximal ideal $\mu \in U_{\lambda}$.

We stress that the open set $U_{\lambda}$ depends on $\lambda$, and often shrinks as $\lambda$ increases.

REMARK 52. Suppose that $f$ has integer coefficients. If $f_{p}$ denotes the image of $f$ in $\mathbb{F}_{p}\left[x_{1}, \ldots, x_{m}\right]$, the statements of Theorem 51 become

$$
\mathbf{f p t}\left(f_{p}\right) \leqslant \mathbf{l c t}(f) \quad \text { for } p \gg 0 \text { and } \quad \lim _{p \rightarrow \infty} \mathbf{f p t}\left(f_{p}\right) \leqslant \mathbf{l c t}(f) \text {. }
$$

Definition 53. Let $f \in S$ be a polynomial vanishing at the origin. We say that log canonicity equals dense (respectively, open) $F$-pure type (at the origin) for $f$ if for every finitely generated $\mathbb{Z}$-algebra $A \subseteq \mathbb{C}$ with $f \in S_{A}$, there exists a dense (respectively, nonempty open) subset $W \subseteq \operatorname{Spec} A$ such that $\mathbf{f p t}\left(f_{\mu}\right)=\operatorname{lct}(f)$ for every maximal ideal $\mu \in W$.

REMARK 54. To show that log canonicity equals (open/dense) F-pure type for $f$, it suffices to produce a single finitely generated $\mathbb{Z}$-algebra $A$ satisfying the conditions of Definition 53. We refer the reader to the author's thesis [Her11] for a detailed verification of this.

REMARK 55. In the study of singularities of pairs, the terms log canonical and $F$-pure have their own independent meanings ${ }^{1}$. Indeed, one defines the notion of $\log$ singularities for pairs $(S, \lambda \bullet f)$ via resolution of singularities (or via integrability conditions) (see [Laz04]). Additionally, we have that

$$
\operatorname{lct}(f)=\sup \{\lambda>0:(S, \lambda \bullet f) \text { is log canonical }\},
$$

which justifies the use of the term log canonical threshold. In the positive characteristic setting, one defines the notion of $F$-purity for pairs via the Frobenius morphism, and we again have that the $F$-pure threshold of a

\footnotetext{
${ }^{1}$ Although we are only interested in the local singularities of polynomials that vanish at $\mathbf{0}$, we will omit the term at the origin, for the sake of clarity.
} 
polynomial is the supremum over all parameters such that the corresponding pair is $F$-pure (see [HW02, TW04]). We say that the pair $(S, \lambda \bullet f)$ is of dense (respectively, open) $F$-pure type (at the origin) if for every (equivalently, for some) finitely generated $\mathbb{Z}$-algebra $A \subseteq \mathbb{C}$ with $f \in S_{A}$, there exists a dense (respectively, nonempty open) set $W \subseteq \operatorname{Spec} A$ such that the pair $\left(S_{A}(\mu), \lambda \bullet f_{\mu}\right)$ is $F$-pure for every maximal ideal $\mu \in W$. It is shown in [HW02, Tak04] that if $(S, \lambda \bullet f)$ is of dense $F$-pure type, then it is also log canonical. It is an important, yet easy to verify, property of $\log$ canonicity that $(S, \operatorname{lct}(f) \bullet f)$ is $\log$ canonical. Consequently, $(S, \lambda \bullet f)$ is $\log$ canonical if and only if $0 \leqslant \lambda \leqslant \operatorname{lct}(f)$. In prime characteristic, that a pair is $F$-pure at the threshold is shown in [Har06, Her12], and it follows that the reductions $\left(S_{A}(\mu), \lambda \bullet f_{\mu}\right)$ are $F$-pure if and only if $0 \leqslant \lambda \leqslant \mathbf{f p t}\left(f_{\mu}\right)$. Examining the definitions, we reach the following conclusion. To show that $\log$ canonicity is equivalent to dense (respectively, open) $F$-pure type for pairs $(S, \lambda \bullet f)$, it suffices to show that $\mathbf{f p t}\left(f_{\mu}\right)=\operatorname{lct}(f)$ for all maximal $\mu$ in some dense (respectively, nonempty open) subset of Spec $A$, which justifies our choice of terminology in Definition 53.

THEOREM 56. Fix a polynomial $f \in S=\mathbb{C}\left[x_{1}, \ldots, x_{m}\right]$ vanishing at the origin, and suppose that the splitting polytope $\mathbf{P}$ associated to $f$ contains a unique maximal point $\boldsymbol{\eta}$. If $A \subseteq \mathbb{C}$ is any finitely generated $\mathbb{Z}$-algebra containing the coefficients of $f$, then the following hold.

(1) If $\operatorname{lct}\left(\mathfrak{a}_{f}\right)>1$, then $\mathbf{f p t}\left(f_{\mu}\right)=\operatorname{lct}(f)=1$ for all $\mu \in \operatorname{mSpec} A$ with char $S_{A}(\mu) \gg 0$. In particular, log canonicity equals open $F$-pure type for $f$.

(2) If $\operatorname{lct}\left(\mathfrak{a}_{f}\right) \leqslant 1$, then for all maximal ideals $\mu \in \mathrm{mSpec} A$ with $\operatorname{char} S_{A}(\mu) \gg 0$,

$$
\operatorname{fpt}\left(f_{\mu}\right)=\operatorname{lct}(f)
$$

whenever the entries of $\boldsymbol{\eta}$ add without carrying (base char $\left.S_{A}(\mu)\right)$. If, in addition, the Newton polyhedron associated to $f$ is in diagonal position, then $\mathbf{f p t}\left(f_{\mu}\right)=\operatorname{lct}(f)$ if and only if the entries of $\boldsymbol{\eta}$ add without carrying (base char $\left.S_{A}(\mu)\right)$. In particular, log canonicity equals dense F-pure type for $f$.

Proof. Let $A$ be such that $f \in S_{A}$, and consider the map $\operatorname{Spec} A \stackrel{\pi}{\rightarrow}$ $\operatorname{Spec} \mathbb{Z}$. After enlarging $A$, we may assume that $\operatorname{Supp}\left(f_{\mu}\right)=\operatorname{Supp}(f)$ for every $\mu \in \operatorname{mSpec} A$, and, hence, that $|\boldsymbol{\eta}|=\operatorname{fpt}\left(a_{f_{\mu}}\right)=\operatorname{lct}\left(\mathfrak{a}_{f}\right)$. First, suppose that $|\boldsymbol{\eta}|>1$. By Lemma 8, there exists a nonempty open subset $U \subseteq \operatorname{Spec} \mathbb{Z}$ 
such that $\eta_{1}{ }^{(1)}+\cdots+\eta_{n}{ }^{(1)} \geqslant p$ for every $p \in U$. For such primes, it follows that $L=0$ in Theorem 42, which then states that $\boldsymbol{f p t}\left(f_{\mu}\right) \geqslant 1$ for every $p \in U$ and $\mu \in \pi^{-1}(U)$. Since $\mathbf{f p t}\left(f_{\mu}\right) \leqslant 1$ always, we see that $1=\mathbf{f p t}\left(f_{\mu}\right) \leqslant$ $\operatorname{lct}(f) \leqslant 1$ for every $\mu \in \pi^{-1}(U)$, which is a nonempty open set, by Corollary 48. We conclude that $\log$ canonicity equals open $F$-pure type for $f$.

Next, suppose that $|\boldsymbol{\eta}| \leqslant 1$, and let $\mathscr{P}$ denote the set of all primes $p$ such that the entries of $\boldsymbol{\eta}$ add without carrying (base $p$ ). By Lemma 8 , the set $\mathscr{P}$ is infinite, and by Corollary $49, \pi^{-1}(\mathscr{P})$ is a dense subset of Spec $A$. Moreover, Theorem 42 states that, if $\operatorname{char} S_{A}(\mu) \gg 0$, then $\boldsymbol{f p t}\left(f_{\mu}\right)=\mathbf{f p t}\left(\mathfrak{a}_{f_{\mu}}\right)$ whenever $\mu \in \pi^{-1}(\mathscr{P})$. Theorem 42 also shows that, if char $S_{A}(\mu) \gg 0$ and $\mathbf{N}_{\mathfrak{a}_{f}}$ is in diagonal position, then $\boldsymbol{f p t}\left(f_{\mu}\right)=\mathbf{f p t}\left(\mathfrak{a}_{f_{\mu}}\right)$ if and only if $\mu \in \pi^{-1}(\mathscr{P})$. Finally, the string of inequalities

$$
\mathbf{f p t}\left(f_{\mu}\right) \leqslant \operatorname{lct}(f) \leqslant \operatorname{lct}\left(\mathfrak{a}_{f}\right)=\mathbf{f p t}\left(\mathfrak{a}_{f_{\mu}}\right)
$$

shows that all of these thresholds agree whenever char $S_{A}(\mu) \gg 0$ and $\mu$ is contained in the dense subset $\pi^{-1}(\mathscr{P})$. We conclude that log canonicity equals dense $F$-pure type for $f$.

LEMMA 57. If $f \in S=\mathbb{C}\left[x_{1}, \ldots, x_{m}\right]$ is a polynomial vanishing at the origin, then log canonicity equals dense $F$-pure type for $f$ if there exist a finitely generated $\mathbb{Z}$-algebra $A \subseteq \mathbb{C}$ with $f \in S_{A}$, an infinite set of primes $\mathscr{P} \subseteq \mathbb{Z}$, and for every $p \in \mathscr{P}$, a subset $\mathscr{D}_{p} \subseteq$ Spec $A$ satisfying the following conditions.

(1) $\mathscr{D}_{p}$ is a dense subset of $\pi^{-1}(p)$, where $\operatorname{Spec} A \stackrel{\pi}{\longrightarrow} \operatorname{Spec} \mathbb{Z}$.

(2) $\mathbf{f p t}\left(f_{\mu}\right)=\min \left\{1, \boldsymbol{f p t}\left(\mathfrak{a}_{f_{\mu}}\right)\right\}$ for every maximal ideal $\mu \in \mathscr{D}_{p}$.

Proof. Let $\mathscr{D}=\bigcup_{p \in \mathscr{P}} \mathscr{D}_{p}$. As $\mathscr{D}_{p}$ is dense in $\pi^{-1}(p), \overline{\mathscr{D}_{p}}=\pi^{-1}(p)$, and thus

$$
\overline{\mathscr{D}} \supseteq \bigcup_{p \in \mathscr{P}} \overline{\mathscr{D}_{p}}=\bigcup_{p \in \mathscr{P}} \pi^{-1}(p)=\pi^{-1}(\mathscr{P}) .
$$

By Corollary $49, \pi^{-1}(\mathscr{P})$ is dense in $\operatorname{Spec} A$, and (6.2) then shows that $\mathscr{D}$ is dense as well. Let $U \subseteq \operatorname{Spec} A$ be the dense open set given by Theorem 51. As $\mathscr{D}$ is dense and $U$ is dense and open, it follows that $U \cap \mathscr{D}$ is dense in $\operatorname{Spec} A$. Furthermore, for $\mu \in U \cap \mathscr{D}$,

$$
\operatorname{lct}(f) \leqslant \min \left\{1, \operatorname{lct}\left(\mathfrak{a}_{f}\right)\right\}=\min \left\{1, \boldsymbol{f p t}\left(\mathfrak{a}_{f_{\mu}}\right)\right\}=\mathbf{f p t}\left(f_{\mu}\right) \leqslant \operatorname{lct}(f) .
$$


Indeed, the leftmost inequality in (6.3) holds by Proposition 45, the first equality by Corollary 46 , the second equality by our assumption on $\mathscr{D}$, and the rightmost inequality by the defining property of $U$. We conclude from (6.3) that $\mathbf{f p t}\left(f_{\mu}\right)=\operatorname{lct}(f)$ for every maximal ideal $\mu$ in the dense subset $U \cap \mathscr{D}$ of $\operatorname{Spec} A$, and the claim follows.

THEOREM 58. Let $f \in \mathbb{C}\left[x_{1}, \ldots, x_{m}\right]$ be a polynomial vanishing at the origin. Fix distinct, nonzero vectors $\mathbf{a}_{1}, \ldots, \mathbf{a}_{n}$ in $\mathbb{N}^{m}$, and elements $u_{1}, \ldots, u_{n}$ in $\mathbb{C}^{*}$, such that $f=\sum_{i=1}^{n} u_{i} x^{\mathbf{a}_{i}}$. If the coefficients $u_{1}, \ldots, u_{n}$ form an algebraically independent sequence over $\mathbb{Q}$, then $\log$ canonicity equals dense $F$-pure type for $f$.

Proof. By hypothesis, $\mathbb{Z}\left[u_{1}, \ldots, u_{n}\right]$ is a polynomial ring, with each $u_{i}$ a variable. If we set $A:=\mathbb{Z}\left[u_{1}, \ldots, u_{n}\right]_{\prod u_{i}} \subseteq \mathbb{C}$, then $f \in S_{A}$, and $\operatorname{Supp}\left(f_{\mu}\right)=$ $\operatorname{Supp}(f)$ for all maximal ideals $\mu \subseteq A$. Set $\gamma=\min \left\{1, \boldsymbol{f p t}\left(\mathfrak{a}_{f_{\mu}}\right)\right\}$, and fix $\boldsymbol{\eta} \in \mathbf{P}_{\max } \cap \mathbb{Q}^{m}$ (such points exist, as $\mathbf{P}$ is rational, and $\mathbf{P}_{\max }$ is a face of $\mathbf{P}$ ). If $\boldsymbol{\lambda}:=\frac{\gamma}{|\boldsymbol{\eta}|} \cdot \boldsymbol{\eta}$, it follows that $\boldsymbol{\lambda} \in P \cap \mathbb{Q}^{m}$ (indeed, it lies in the line segment connecting $\mathbf{0}$ and $\boldsymbol{\eta}$ ), and satisfies $|\boldsymbol{\lambda}|=\gamma$. If $\mathscr{P}$ denotes the set of primes $p$ such that $(p-1) \cdot \boldsymbol{\lambda} \in \mathbb{N}^{n}$, then $\# \mathscr{P}=\infty$ by Dirichlet's theorem on primes in arithmetic progressions. Fix a prime $p \in \mathscr{P}$. It follows from the multinomial theorem that $x^{(p-1) \mathbf{E} \boldsymbol{\lambda}}$ appears in $f^{(p-1) \gamma}$ with coefficient

$$
\Theta_{\boldsymbol{\lambda}, p}\left(u_{1}, \ldots, u_{n}\right)=\sum\left(\begin{array}{c}
(p-1) \cdot \alpha \\
\mathbf{k}
\end{array}\right) u^{\mathbf{k}} \in \mathbb{Z}\left[u_{1}, \ldots, u_{n}\right] \subseteq A,
$$

where the sum extends over all $\mathbf{k} \in \mathbb{N}^{n}$ such that $|\mathbf{k}|=(p-1) \gamma$, and $\mathbf{E k}=$ $(p-1) \cdot \mathbf{E} \boldsymbol{\lambda}$. As $(p-1) \cdot \boldsymbol{\lambda}$ is such an index, it follows that $\Theta_{\boldsymbol{\lambda}, p}\left(u_{1}, \ldots, u_{n}\right)$ defines a nonzero element of the polynomial ring $\mathbb{Z}\left[u_{1}, \ldots, u_{n}\right]$. Moreover, as $\gamma \leqslant 1,\left(\begin{array}{c}(p-1) \cdot \gamma \\ \mathbf{k}\end{array}\right) \neq 0 \bmod p$ for each $\mathbf{k}$ in (6.4), and it follows that the image of $\Theta_{\boldsymbol{\lambda}, p}\left(u_{1}, \ldots, u_{n}\right)$ defines a nonzero element of the polynomial ring $\mathbb{Z} / p \mathbb{Z}\left[u_{1}, \ldots, u_{n}\right] \subseteq A / p A$. Consider the map Spec $A \stackrel{\pi}{\rightarrow}$ Spec $\mathbb{Z}$ induced by the inclusion $\mathbb{Z} \subseteq A$. We have just shown that $\mathscr{D}_{p}:=D\left(\Theta_{\boldsymbol{\lambda}, p}(\mathbf{u})\right) \cap \pi^{-1}(p)$ is a dense (and, in fact, open) subset of the fiber $\pi^{-1}(p)$. Let $\mu_{p}$ be a maximal ideal in $\mathscr{D}_{p}$. By definition, the image of $\Theta_{\boldsymbol{\lambda}, p}(\mathbf{u})$ is nonzero in $A / \mu_{p}$, and (6.4) shows that $\boldsymbol{x}^{(p-1) \mathbf{E} \boldsymbol{\lambda}}$ is contained in $\operatorname{Supp}\left(\left(f_{\mu_{p}}\right)^{(p-1) \gamma}\right)$ but not in $\mathfrak{m}^{[p]}$ (as $\boldsymbol{\lambda} \in \mathbf{P})$. Thus, $\left(f_{\mu_{p}}\right)^{(p-1) \gamma} \notin \mathfrak{m}^{[p]}$, which allows us to apply Lemma 13 to $f_{\mu_{p}} \in S_{A}\left(\mu_{p}\right)$ to conclude that $\mathbf{f p t}\left(f_{\mu_{p}}\right) \geqslant \gamma=\min \left\{1, \boldsymbol{f p t}\left(\mathfrak{a}_{f_{\mu}}\right)\right\}$. By Remark 12 , the opposite bound always holds, and we conclude that $\operatorname{fpt}\left(f_{\mu_{p}}\right)=$ $\min \left\{1, \boldsymbol{f p t}\left(\mathfrak{a}_{f_{\mu_{p}}}\right)\right\}$ for every $p \in \mathscr{P}$, and $\mu_{p} \in \mathscr{D}_{p}$. In conclusion, we see that $A, \mathscr{P}$, and $\mathscr{D}_{p}$ satisfy the conditions of Lemma 57 , and so we are done. 
Acknowledgments. Many of the results contained in this article appear in the author's Ph.D. thesis, completed at the University of Michigan. The author would like to thank his advisor, Karen Smith, for her guidance during this project. The author would also like to thank Emily Witt, Karl Schwede, Mel Hochster, and Vic Reiner for their useful comments on earlier versions of this article.

\section{REFERENCES}

[BFS13] A. Benito, E. Faber and K. E. Smith, "Measuring singularities with Frobenius: the basics", in Commutative Algebra, Springer, New York, 2013, 57-97.

[BL04] M. Blickle and R. Lazarsfeld, "An informal introduction to multiplier ideals", in Trends in Commutative Algebra, Math. Sci. Res. Inst. Publ. 51, Cambridge University Press, Cambridge, 2004, 87-114.

[BMS08] M. Blickle, M. Mustaţă and K. E. Smith, Discreteness and rationality of Fthresholds, Michigan Math. J. 57 (2008), 43-61; Special volume in honor of Melvin Hochster.

[BMS09] M. Blickle, M. Mustaţă and K. E. Smith, F-thresholds of hypersurfaces, Trans. Amer. Math. Soc. 361(12) (2009), 6549-6565.

[Dic02] L. E. Dickson, Theorems on the residues of multinomial coefficients with respect to a prime modulus, Quart. J. Pure Appl. Math. 33 (1902), 378-384.

[EM06] L. Ein and M. Mustaţă , "Invariants of singularities of pairs", in International Congress of Mathematicians. Vol. II, European Mathematical Society, Zürich, 2006, 583-602.

[Fed83] R. Fedder, F-purity and rational singularity, Trans. Amer. Math. Soc. 278(2) (1983), 461-480.

[Har06] N. Hara, F-pure thresholds and F-jumping exponents in dimension two, Math. Res. Lett. 13(5-6) (2006), 747-760; with an appendix by Paul Monsky.

[Her11] D. J. Hernández, F-purity of hypersurfaces. Ph.D. thesis, University of Michigan, http://hdl.handle.net/2027.42/86491, 2011.

[Her12] D. J. Hernández, F-purity of hypersurfaces, Math. Res. Lett. 19(02) (2012), $1-13$.

[Her14] D. J. Hernández, F-pure thresholds of binomial hypersurfaces, Proc. Amer. Math. Soc. 142(7) (2014), 2227-2242.

[Her15] D. J. Hernández, F-invariants of diagonal hypersurfaces, Proc. Amer. Math. Soc. 143(1) (2015), 87-104.

[HH90] M. Hochster and C. Huneke, Tight closure, invariant theory, and the BriançonSkoda theorem, J. Amer. Math. Soc. 3(1) (1990), 31-116.

[How01a] J. A. Howald, Multiplier ideals of monomial ideals, Trans. Amer. Math. Soc. 353(7) (2001), 2665-2671; (electronic).

[How01b] J. Howald, Multiplier ideals of sufficiently general polynomials. preprint, math.AG/0303203, 2001.

[HR76] M. Hochster and J. L. Roberts, The purity of the Frobenius and local cohomology, Adv. Math. 21(2) (1976), 117-172.

[HW02] N. Hara and K.-I. Watanabe, F-regular and F-pure rings vs. log terminal and log canonical singularities, J. Algebraic Geom. 11(2) (2002), 363-392.

[HY03] N. Hara and K.-I. Yoshida, A generalization of tight closure and multiplier ideals, Trans. Amer. Math. Soc. 355(8) (2003), 3143-3174; (electronic). 
[Laz04] R. Lazarsfeld, Positivity in Algebraic Geometry. II, Ergebnisse der Mathematik und ihrer Grenzgebiete. 3. Folge. A Series of Modern Surveys in Mathematics [Results in Mathematics and Related Areas. 3rd Series. A Series of Modern Surveys in Mathematics] 49, Springer, Berlin, 2004, Positivity for vector bundles, and multiplier ideals.

[Luc78] E. Lucas, Theorie des Fonctions Numeriques Simplement Periodiques (French), Amer. J. Math. 1(2) (1878), 184-196.

[MTW05] M. Mustaţă, S. Takagi and K.-i. Watanabe, "F-thresholds and Bernstein-Sato polynomials", in European Congress of Mathematics, European Mathematical Society, Zürich, 2005, 341-364.

[Sch08] K. Schwede, Generalized test ideals, sharp F-purity, and sharp test elements, Math. Res. Lett. 15(6) (2008), 1251-1261.

[Smi97] K. E. Smith, "Vanishing, singularities and effective bounds via prime characteristic local algebra", in Algebraic Geometry - Santa Cruz 1995, Proc. Sympos. Pure Math. 62, American Mathematical Society, Providence, RI, 1997, 289-325.

[Smi00] K. E. Smith, The multiplier ideal is a universal test ideal, Comm. Algebra 28(12) (2000), 5915-5929; special issue in honor of Robin Hartshorne.

[ST09] T. Shibuta and S. Takagi, Log canonical thresholds of binomial ideals, manuscripta mathematica 130(1) (2009), 45-61.

[Tak04] S. Takagi, F-singularities of pairs and inversion of adjunction of arbitrary codimension, Invent. Math. 157(1) (2004), 123-146.

[TW04] S. Takagi and K.-i. Watanabe, On F-pure thresholds, J. Algebra 282(1) (2004), $278-297$.

[Web94] R. Webster, Convexity, Oxford Science Publications, The Clarendon Press Oxford University Press, New York, 1994.

University of Utah

Math Department: 155 S 1400 E Room 233

Salt Lake City

UT 84112-0090

USA

dhernan@math.utah.edu 